\title{
Matching to Share Risk without Commitment*
}

\author{
Johannes Gierlinger ${ }^{\dagger}$ and Sarolta Laczó ${ }^{\ddagger}$
}

January 2017

\begin{abstract}
This paper studies the effect of limited commitment on sorting when two sides of a frictionless market form pairs to share risk. On each side, agents are identical except for their risk preferences. First, we provide analytical results when transfers do not condition on the history of shocks. More risk-averse agents can commit to larger transfers, as long as their consumption is less risky than their endowment. With sufficiently large idiosyncratic risk and sufficient discounting of the future, matching is positive assortative, unlike under full commitment. Second, we find positive-assortative stable matchings when transfers are history dependent using a numerical algorithm.
\end{abstract}

Keywords: assortative matching, imperfectly transferable utility, risk sharing, limited commitment

JEL codes: D86, C73, C78.

${ }^{*}$ We thank the editor Martin Cripps, two anonymous referees, and seminar and conference participants at Universitat Autònoma de Barcelona, Institut d'Anàlisi Econòmica, ESEM in Oslo, ENTER Jamboree in Brussels, Barcelona GSE Summer Forum, CEF in Vancouver, UIMP, RES in Manchester, EEA in Toulouse, University of Alicante, and the Coalition Theory Network workshop in Moscow for useful comments and suggestions. All errors are our own. Gierlinger gratefully acknowledges financial support from the Spanish Ministry of Economy and Competitiveness through grant ECO2015-67602-P and through the Severo Ochoa Programme for Centres of Excellence in R\&D (SEV-2011-0075). The research leading to this paper received funding from the European Research Council under the European Community's Seventh Framework Programme (FP7/2007-2013) Grant agreement no 230589. Financial support by Fundación Ramón Areces is gratefully acknowledged. Laczó acknowledges funding from the Spanish Ministry of Science and Innovation, grant ECO2008-04785, and the JAE-Doc programme co-financed by the European Social Fund.

†Universitat Autònoma de Barcelona-MOVE and Barcelona GSE, Departament d'Economia i d'Història Econòmica, Edifici B, 08193 Bellaterra, Barcelona, Spain. Email: johannes.gierlinger@uab.es

${ }^{\ddagger}$ University of Surrey, IAE-CSIC, and CEPR, School of Economics, AD Building ground floor, Guildford, Surrey, GU2 7XH, United Kingdom. Email: s.laczo@surrey.ac.uk. 


\section{Introduction}

Consider a two-sided, frictionless matching market whose members, 'men' and 'women,' are risk averse, and who may form 'couples' to share an exogenous risk. Agents are heterogeneous in their risk attitudes, but each man and woman faces the same gender-specific, non-tradable risk. In short, consider a marriage problem with imperfectly transferable utility and without search frictions. Legros and Newman (2007) and Chiappori and Reny (2016) show that all stable matchings are negative assortative in risk aversion in the Arrow-Pratt sense if couples are assumed to share risk efficiently. That is, for any two couples, the more risk-averse man is matched with the less risk-averse woman. Efficient risk sharing generates the greatest surplus in diverse couples. The more risk-averse man is willing to decrease his consumption share more in return for insurance. This makes him an attractive partner for the less risk-averse woman, who has a comparative advantage in providing insurance.

The novelty in this paper is to acknowledge possible commitment problems. Our goal is to study whether the negative-assortative-matching prediction in the literature (Legros and Newman, 2007; Chiappori and Reny, 2016) is robust to limited commitment. To do so, we build on the literature on risk sharing with self-enforcing transfers (Thomas and Worrall, 1988; Kocherlakota, 1996). In addition to ex-ante participation constraints, where each 'spouse' requires at least the utility he or she would get when 'single,' the contract must satisfy ex-post participation constraints. That is, for any history of endowment realisations, the sharing rule must be sustainable by voluntary transfers.

In our model matching takes place at time 0 , and risk sharing takes place from time 1 onwards. ${ }^{1}$ First, we consider self-enforcing sharing rules with static contracts, which do not condition on past realisations of shocks, as in Coate and Ravallion (1993). This is a natural generalisation of atemporal models which admits analytical solutions. In addition, we follow Legros and Newman (2007) in studying the case of a binary state space for analytical tractability.

In contrast to full commitment, we find that sorting depends on the correlation of endowments, or, more precisely, on the relative magnitude of idiosyncratic and aggregate risk. We show that (i) if endowments covary negatively, then all stable matchings are positive assortative for low enough discount factors, that is, when the commitment problem is sufficiently severe. The threshold discount factor depends on the relative magnitude of idiosyncratic risk and aggregate risk. On the other hand, (ii) if the correlation of endowments is non-negative,

\footnotetext{
${ }^{1}$ Recent literature on dynamic matching, such as Anderson and Smith (2010), considers (re)matching at any time. Our qualitative results are robust to rematching if finding a new partner takes time, as discussed at the end of Section 3.1.
} 
then matching is always negative assortative.

The intuition behind these results is the following. A key determinant of sorting is whether risk aversion helps commitment on both sides of the market. This in turn depends on whether consumption after risk-sharing transfers is less risky than the endowment for both spouses. Risk aversion helps an agent to commit to transfers which reduce his/her own consumption risk relative to autarky, while it makes taking on additional risk less credible. The two sides can mutually reduce their consumption risk through transfers only when endowments are negatively correlated. In this case, more risk-averse men and women can credibly commit to larger transfers and hence achieve more insurance. If the discount factor is sufficiently low, this effect dominates the effect of more efficient sharing of uninsurable risk among more diverse agents, hence stable matchings are positive assortative. Instead when endowments are positively correlated, an agent's consumption risk can only be reduced at the expense of increasing the partner's risk. In this case, risk aversion makes the commitment problem less severe on one side of the market and more severe on the other. Hence couples with more diverse risk preferences achieve more insurance, and matching is negative assortative.

Finally, we study whether our results are robust to history-dependent contracts (see Kocherlakota, 1996, and others). While the long-run allocations can be characterised analytically, sorting also depends on the couple's ability to sustain transfers during an initial transition period. We turn to numerical methods and propose an algorithm to identify stable matchings and determine their assortativity. We confirm that stable matchings can be positive assortative allowing for history-dependent sharing rules as well.

Two-sided, one-to-one matching with imperfectly transferable utility is relevant in a wide range of applications, including household formation, principal-agent models, e.g. matching projects to managers (see Legros and Newman, 2007), matching workers to firms or into teams (pairs with complementary skills), joint ventures, and sharecropping agreements. Further, limited commitment is a relevant friction in all these applications, because interaction is likely to occur repeatedly once a match is formed, and quitting is an option. We discuss two applications in more detail, household formation and matching workers to firms.

The risk-sharing motive for household formation has been highlighted in several papers, including Kotlikoff and Spivak (1981), Rosenzweig and Stark (1989), Ogaki and Zhang (2001), and Hess (2004). Recent literature investigates whether the risk-sharing motive might help to explain the composition of households, see Schulhofer-Wohl (2006), Legros and Newman (2007), and Chiappori and Reny (2016). Compared to negative-assortative matching, positive-assortative matching reduces heterogeneity within couples and increases heterogeneity across couples. The answer to the question whether risk preferences within the households 
are similar is key to link individual preferences with decisions at the household level, such as consumption-saving (Mazzocco, 2004) and portfolio allocation. Moreover, much of the empirical literature builds on the assumption of identical preferences within the household (see Chiappori and Reny, 2016, for a discussion). If matching were indeed negative assortative, then such an assumption would be problematic. Furthermore, empirical evidence suggests that limited commitment is relevant within households. Using the Consumer Expenditure Survey (CEX), Mazzocco (2007) finds inter-temporal decisions within US households to be subject to limited commitment. Similarly, Dercon and Krishnan (2000) provide evidence against efficient risk sharing within households in rural Ethiopia.

Alternatively, consider firms matching with workers. Both sides are risk averse and heterogeneous in their risk aversion. ${ }^{2}$ For a matched firm-worker pair, the wage is determined according to a long-term wage contract as in Thomas and Worrall (1988). ${ }^{3}$ In this paper we ask whether more risk-averse workers sort into jobs where the employer is more risk averse. Risk-sharing considerations in wage determination have been highlighted by Guiso, Pistaferri, and Schivardi (2005), who find that shocks to a firm's output can account for about 15 percent of the overall variability of workers' earnings. Further, building on Thomas and Worrall (1988), recent literature suggests that limited commitment may explain the observed wage rigidity in long-term contracts on the US labour market, see Rudanko (2009).

In this paper we follow Legros and Newman (2007) and Chiappori and Reny (2016) in restricting our attention to economies where matching is fully determined by risk sharing. Recent literature shows that matching under full commitment may no longer be negative assortative in risk aversion if spouses jointly decide on the riskiness of their income as well. ${ }^{4}$ Indeed, homogeneous spouses may find it easier to agree on their joint exposure to risk. On the other hand, the efficient sharing of any such risk still favours spouses who are heterogeneous, and sorting depends on which of the two forces dominates. Our results suggest that even if risk sharing determines sorting, matching may still be positive assortative if

\footnotetext{
${ }^{2}$ A large literature, starting with the seminal work of Sandmo (1971), analyses the behaviour of risk-averse firms.

${ }^{3}$ Note that we deviate from Thomas and Worrall (1988) in assuming that the firm is risk averse.

${ }^{4}$ Wang (2015) considers couples who select a joint project according to risk and return. He finds that matching is positive assortative if the risk in higher return projects increases at a growing rate, as measured by an increasing coefficient of variation. In this case, agents find it optimal to match along their preferences over projects. Conversely, if the coefficient of variation is decreasing in expected return, the gains from efficient risk sharing dominate, and matching is negative assortative. In Li, Sun, and Chen (2013), the two sides may reduce household risk by exerting joint effort. Matching is positive assortative in settings of either high risk aversion, or when effort has a sufficiently large effect on income risk. Serfes (2005) considers both endogenous income and moral hazard. Risk-neutral principals who differ in the riskiness of their projects are matched with agents who differ in risk attitudes and whose effort is unobservable. He finds that the equilibrium relationship between risk and incentives can be both positive and negative.
} 
commitment plays a role.

The rest of the paper is structured as follows. Section 2 details the economic environment, and characterises equilibria under the benchmark assumption of full commitment. Section 3 contains the main results of the paper, providing sufficient condition for assortativity of stable matchings without commitment. We first consider static risk-sharing contracts (Section 3.1), then extend our analysis to dynamic contracts (Section 3.2). Section 4 concludes. Appendices contain the proofs (Appendix A), results on the pattern of binding participation constraints (Appendix B), and our algorithm to find stable matchings and determine their assortativity with dynamic contracts (Appendix C).

\section{The environment}

Consider an endowment economy with $2 N$ infinitely lived agents, each belonging to one of two disjoint sub-populations, $\mathcal{I}$ and $\mathcal{J}$ of equal cardinality. From here onwards, we refer to members $i \in \mathcal{I}$ and $j \in \mathcal{J}$ as 'women' and 'men', respectively. However, our framework accommodates a host of interpretations other than potential spouses, including firms and workers who may enter a long-term relationship.

Agents on each side of the matching market are exposed to an exogenous income risk at each date $t=1,2, \ldots$, which is independently and identically distributed (i.i.d.) over time. The income process is gender-specific, but not specific to any individual. Let $s_{t} \in\{\bar{s}, \underline{s}\}$ denote the state of the world at time $t$ that governs both joint and individual levels of endowment in terms of the perishable consumption good. For analytical tractability, we consider a binary state space, as in Legros and Newman (2007). Both this and the i.i.d. assumption can be relaxed in our numerical analysis. In particular, we can accommodate any Markovian endowment process with finite state space.

The vector $W=(\bar{w}, \underline{w})$ denotes women's state-contingent income, $\bar{w}$ and $\underline{w}$ in states $\bar{s}$ and $\underline{s}$, respectively. Similarly, we denote men's income by $M=(\bar{m}, \underline{m})$. The state probabilities $\operatorname{Pr}\left(s_{t}=\underline{s}\right)=\pi$ and $\operatorname{Pr}\left(s_{t}=\bar{s}\right)=1-\pi$, as well as the income vectors are common knowledge. If Ms $i$ and $\mathrm{Mr} j$ decide to form a couple, their aggregate endowment is therefore $Z=W+M=(\bar{z}, \underline{z})$. Without loss of generality, we assume $\bar{z} \geq \underline{z}$.

The instantaneous utility function of Ms $i$ is denoted $u(i, c)$, where $c$ denotes consumption. ${ }^{5}$ We assume that $u$ is twice differentiable in consumption and that it exhibits strict

\footnotetext{
${ }^{5} u(i, \cdot)$ can be interpreted as an indirect utility function which captures the attitude toward the residual uninsurable risk in the presence of otherwise competitive financial markets. If the tradable risk is statistically independent from the uninsurable risk, then $u(i, \cdot)$ inherits monotonicity and risk aversion from utility over total wealth (see Gollier, 2001).
} 
monotonicity and strict risk aversion, i.e., $u_{c} \equiv \partial u / \partial c>0$ and $u_{c c} \equiv \partial^{2} u / \partial c^{2}<0$ everywhere. We assume further that $u$ satisfies the Inada conditions with respect to $c$ for all agents, i.e., $\lim _{c \rightarrow 0} u_{c}(i, c)=+\infty$, and $\lim _{c \rightarrow+\infty} u_{c}(i, c)=0$. Finally, we denote expected per-period utility from a state-contingent consumption vector $C=(\bar{c}, \underline{c})$ by

$$
\mathbb{E} u(i, C)=(1-\pi) u(i, \bar{c})+\pi u(i, \underline{c}) .
$$

Alternatively, in our second application, substituting workers for women and firms for men, we call $W$ the exogenous and stochastic market wage (one unit of labour is the only production input) and $Z$ the firm's revenue. If the firm hires a unit of labour on the spot market, then its profit is $M=Z-W$. Firms and workers can instead match in order to agree on a long-term state-contingent wage contract $C$.

Individuals are heterogenous with respect to their risk preferences. We assume throughout this paper that Ms $i^{\prime \prime}$ is strictly more risk averse than Ms $i^{\prime}$ in the Arrow-Pratt sense. That is, there exists a strictly increasing and strictly concave function $\phi$ which satisfies $u\left(i^{\prime \prime}, \cdot\right)=\phi\left(u\left(i^{\prime}, \cdot\right)\right)$. Analogously, we assume that $\operatorname{Mr} j^{\prime \prime}$ is strictly more risk averse than Mr $j^{\prime}$.

The timing of agents' interaction is as follows. At date $t=0$, the two sides of the market meet to form couples. At any subsequent date $t=1,2, \ldots$, nature first draws the publicly observable state, then matched agents may decide to transfer some of their current income to their partner, and finally consumption takes place.

Our definitions of stability and assortative matching are standard. Define the assignment functions $\mu: \mathcal{I} \rightarrow\{0\} \cup \mathcal{J}$ and $\psi: \mathcal{J} \rightarrow\{0\} \cup \mathcal{I}$ as follows: if Ms $i(\operatorname{Mr} j)$ is single, then $\mu(i)=0(\psi(j)=0)$ and we write $\langle i\rangle(\langle j\rangle)$. Any matched couple, denoted $\langle i, j\rangle$, satisfies $\mu(i)=j$ and $\psi(j)=i$. Finally, let $\succsim_{i}\left(\succ_{i}\right)$ be Ms $i$ 's weak (strict) ranking over potential partners $\{0\} \cup \mathcal{J}$, that is, $j \succsim_{i} \tilde{j}\left(j \succ_{i} \tilde{j}\right)$ means that Ms $i$ weakly (strictly) prefers Mr $j$ over $\operatorname{Mr} \tilde{j}$. Similarly, let $\succsim_{j}\left(\succ_{j}\right)$ be the analogous ranking over $\{0\} \cup \mathcal{I}$ for $\operatorname{Mr} j$.

Definition 1 (Stable matching). A couple $\langle i, j\rangle$ is individually rational if $j \succsim_{i} 0$ and $i \succsim_{j} 0$, i.e., no agent strictly prefers to be single. A couple $\langle i, j\rangle$ is a blocking pair if $j \succ_{i} \mu(i)$ and $i \succ_{j} \psi(j)$. A matching is stable, if all couples are individually rational and if no blocking pair exists.

Definition 2 (Assortative matching). Consider any two couples $j=\mu(i)$ and $\tilde{j}=\mu(\tilde{i})$. A matching is positive assortative (PA) if $j>\tilde{j} \Rightarrow i>\tilde{i}$. A matching is negative assortative (NA) if $j>\tilde{j} \Rightarrow i<\tilde{i}$. A matching is payoff equivalent to an alternative matching if it preserves every agent's expected discounted life-time utility. We say that the economy 
satisfies PAM (NAM) if every stable matching is payoff equivalent to a positive (negative) assortative matching.

Notice that PAM and NAM are not mutually exclusive. There may exist a payoff-equivalent PA matching to every NA matching, and vice versa.

Below, we make frequent use of two properties which help us organise and compare alternative consumption rules. First, call $\mathbf{C}^{+} \equiv\{(\bar{c}, \underline{c}): \bar{c} \geq \underline{c}\}$ the set of state-contingent payoffs which prescribe (weakly) higher consumption when joint income is high. Similarly, define $\mathbf{C}^{-} \equiv\{(\bar{c}, \underline{c}): \bar{c} \leq \underline{c}\}$. Second, we use the following single-crossing criterion due to Jewitt (1986) to identify when two agents with diverse risk preferences may disagree on the ranking of risky prospects.

Definition 3 (Jewitt's order). A random variable $A \sim F_{A}$ dominates $B \sim F_{B}$ according to Jewitt's order, $A \succsim_{S C} B$, if there exists a value $x_{0}$ such that for all $x$,

$$
\left(x-x_{0}\right)\left(F_{A}(x)-F_{B}(x)\right) \geq 0 .
$$

In this case we also say that $A$ is less Jewitt-risky than $B$.

Consider Ms $i^{\prime \prime}$ and Ms $i^{\prime}$, and recall that Ms $i^{\prime \prime}$ is more risk averse than Ms $i^{\prime}$. Jewitt (1986) shows that the relation $A \succsim_{S C} B$ characterises the following behavioural property: if Ms $i^{\prime}$ prefers a random variable $A$ over $B$, then so does Ms $i^{\prime \prime}$, i.e., $A \succsim_{i^{\prime}} B \Rightarrow A \succsim_{i^{\prime \prime}} B$. Conversely, if Ms $i^{\prime \prime}$ prefers $B$ over $A$, then so does Ms $i^{\prime}$.

With a binary state space, if $F_{A}$ crosses $F_{B}$ from below, then $A$ must be a reduced spread of $B$, i.e., $\bar{b} \geq \bar{a} \geq \underline{a} \geq \underline{b}$. Note that if $A$ and $B$ belong to $\mathbf{C}^{+}$(or if both belong to $\mathbf{C}^{-}$), then $F_{A}$ and $F_{B}$ cannot cross more than once. If they do not cross at all, then $A$ and $B$ can be ranked by first-degree stochastic dominance (FSD).

Finally, we draw on sufficient conditions for assortative matching due to Legros and Newman (2007). Let $C_{i j}(v)$ denote the state-contingent consumption vector of Ms $i$ when sharing risk with $\mathrm{Mr} j$, given a feasible utility promise $v$ to $\mathrm{Mr} j$. The economy satisfies NAM if

$$
C_{i^{\prime \prime} j^{\prime \prime}}\left(v^{\prime \prime}\right) \succsim_{i^{\prime \prime}} C_{i^{\prime \prime} j^{\prime}}\left(v^{\prime}\right) \Rightarrow C_{i^{\prime} j^{\prime \prime}}\left(v^{\prime \prime}\right) \succsim_{i^{\prime}} C_{i^{\prime} j^{\prime}}\left(v^{\prime}\right),
$$

where $C \succsim_{i} D$ if and only if the expected lifetime utility of Ms $i$ is greater under contract $C$ than under D. Condition GDD is a generalised decreasing difference condition: given utility promises $v^{\prime}$ and $v^{\prime \prime}$, if the more risk-averse woman Ms $i^{\prime \prime}$ prefers to match with the more risk-averse man $\mathrm{Mr} j^{\prime \prime}$, so does the less risk-averse woman. For GDD to hold, less risk-averse 
women should have their comparative advantage in sharing risk with more risk-averse men. Similarly, the following generalised increasing difference (GID) condition guarantees PAM:

$$
C_{i^{\prime} j^{\prime \prime}}\left(v^{\prime \prime}\right) \succsim_{i^{\prime}} C_{i^{\prime} j^{\prime}}\left(v^{\prime}\right) \quad \Rightarrow \quad C_{i^{\prime \prime} j^{\prime \prime}}\left(v^{\prime \prime}\right) \succsim_{i^{\prime \prime}} C_{i^{\prime \prime} j^{\prime}}\left(v^{\prime}\right)
$$

In order to distinguish the effects of repeated interaction from those of limited commitment, we review the full commitment benchmark by considering the infinitely repeated extension of the one-shot game in the literature.

\subsection{Sorting under full commitment}

To fix ideas, consider first a matched couple's ex-ante problem for a single period. If Ms $i$ promises expected utility $v$ to her partner $\operatorname{Mr} j$, then the corresponding optimal sharing rule, denoted $C_{i j}^{*}(v)=\left(\bar{c}_{i j}^{*}(v), \underline{c}_{i j}^{*}(v)\right)$, must satisfy

$$
C_{i j}^{*}(v) \equiv \arg \max _{C} \mathbb{E} u(i, C) \quad \text { s.t. } \quad \mathbb{E} u(j, Z-C) \geq v .
$$

Varying $v$, the solutions $C_{i j}^{*}(v)$ describe the set of efficient sharing rules. Let $x(i, C)$ denote the marginal rate of substitution (MRS) between the two states for agent $i$, i.e.,

$$
x(i, C) \equiv \frac{u_{c}(i, \bar{c})}{u_{c}(i, \underline{c})} .
$$

Thanks to our regularity assumptions on the utility functions, it is optimal to equate marginal rates of substitution. That is, we have $x\left(i, C_{i j}^{*}(v)\right)=x\left(j, Z-C_{i j}^{*}(v)\right)$ (Borch, 1962).

Consider now the case where risk sharing takes place over infinitely many periods. Given that preferences are separable over time with a common discount factor, we recover the classic result that ratios of marginal utility remain constant across time and states. The

efficient many-period sharing rule prescribes the one-period arrangement $C_{i j}^{*}(v)$ from (1) in each period $t$, where $v$ is the per-period expected utility associated to a lifetime-utility promise $v /(1-\beta)$.

Taking into account the woman's expected lifetime utility if she promises $v$ to $\mathrm{Mr} j$, we apply the sufficient condition for NAM in Legros and Newman (2007). Accordingly, our economy satisfies NAM if, for any feasible utility levels $v^{\prime}=\mathbb{E} u\left(j^{\prime}, Z-C\right)$ and $v^{\prime \prime}=\mathbb{E} u\left(j^{\prime \prime}, Z-C\right)$, GDD holds. We use Jewitt's order to provide an alternative proof for the NAM result in the literature (Legros and Newman, 2007; Chiappori and Reny, 2016), which may be of independent interest for assortative matching among risk-averse agents. In contrast to the previous literature, we allow for the case where joint income does not depend on the state, i.e., $\bar{z}=\underline{z}$.

Proposition 1. Under full commitment, the economy satisfies NAM. 
Proof. In Appendix A.

The intuition behind this result is as follows. Efficient risk sharing must eliminate all idiosyncratic risk in every couple. As a result, consumption after transfers is an increasing function of joint income. The spouses' risk attitudes only affect the allocation of aggregate (not mutually insurable) risk. Whenever a woman accepts additional aggregate risk, the effect on the partner's expected utility will be greater if he is more risk averse. Similarly, if a woman is less risk averse, then she has a comparative advantage in bearing additional aggregate risk. Given that idiosyncratic risk does not matter in equilibrium, the ranking of partners on each side is fully determined by the couple's ability to generate surplus from sharing aggregate risk. Therefore, since diversity in risk preferences increases the joint surplus, we obtain GDD. Note that the special case where joint income is constant $(\bar{z}=\underline{z})$ guarantees constant consumption in all couples. As a result, any two women rank any two men identically according to the promised level of constant consumption. Hence both GDD and GID hold in this case, and we have both NAM and PAM.

\section{Sorting under limited commitment}

We now ask whether commitment problems have a systematic effect on the sorting properties of stable matchings with respect to risk aversion, i.e., we relax the assumption of full commitment in Legros and Newman (2007) and Chiappori and Reny (2016). We assume that insurance transfers must be self-enforcing in the sense of Thomas and Worrall (1988) and Kocherlakota (1996). That is, the expected lifetime utility of each spouse must be at least the autarky level at each time and state. Following these seminal papers, we assume that agents apply a trigger strategy to enforce their partner's promises: defectors can be excluded from any future risk-sharing arrangements, and they consume their autarky endowment process. This is the most severe subgame-perfect punishment for defection in this environment (Kocherlakota, 1996). That is, agents have access to an optimal penal code in the sense of Abreu (1988) to help them sustain cooperation. However, as we discuss in more detail below, our results extend to various alternative specifications of the outside option, both exogenous and endogenous.

Lack of commitment restricts the set of credible utility promises in period 0. In particular, couples may be unable to sustain efficient risk sharing, and the solution for each couple depends not only on the risk preferences of both spouses and the utility promise $v$ to the man, but also the discount factor $\beta$. 
In addition, as we show below, sorting under limited commitment depends on the correlation of incomes on the two sides of the market, or, more precisely, on the relative importance of idiosyncratic and aggregate risk. In order to take this into account, we parameterise the income processes as follows. We assume, without loss of generality, that men's endowment satisfies $M=(\bar{m}, \underline{m})=(1+y, 1-y)$, where $y \in(0,1) .{ }^{6}$ Further, we assume that women's endowment takes the values $W=(\bar{w}, \underline{w})=(1+\varepsilon y, 1-\varepsilon y)$, where $\varepsilon \in[-1,1] .^{7}$ If $\varepsilon=-1$, the couple faces a risk-free joint income and only idiosyncratic risk. On the other hand, with $\varepsilon=1$ there is only aggregate risk, and any gains from sharing risk must be due to heterogeneous risk attitudes. In this case the coefficient of variation of the aggregate endowment is $y$. With $\varepsilon=0$, women face no income risk, and incomes are uncorrelated. As $\varepsilon$ increases from -1 to 1 , the coefficient of variation of aggregate income increases from 0 to $y$, while idiosyncratic (or, zero-sum) risk decreases. Hence, we can say that the magnitude of aggregate risk relative to idiosyncratic risk increases with $\varepsilon .^{8}$

In our second application, to conform with our assumption of limited commitment, we assume that both workers and firms may unilaterally terminate the long-term wage contract at any time having observed the current market wage, and find (an) alternative work(er) at that wage rate in the same period, as in Thomas and Worrall (1988). The correlation between $M$ and $W$ captures if and how the firm's revenue $Z$ correlates with the stochastic market wage rate.

Before analysing history-dependent contracts in Section 3.2, we first consider the effect of commitment on sorting when sharing rules do not condition on past shocks, as in Coate and Ravallion (1993). This allows us to determine analytically how risk aversion affects both the commitment problem and the sharing rule, and in turn to provide sufficient conditions for assortative matching.

\subsection{Static contracts}

Call $C_{i j}(v ; \beta, \varepsilon)=\left(\bar{c}_{i j}(v ; \beta, \varepsilon), \underline{c}_{i j}(v ; \beta, \varepsilon)\right)$ the static sharing rule which maximises the utility of Ms $i$ subject to the participation constraints and the expected per-period utility promise

\footnotetext{
${ }^{6}$ Note that our formulation can accommodate different mean incomes on the two sides of the market by a normalisation of the utility functions.

${ }^{7}$ The cases where women's risk is higher can be nested by appropriate relabelling.

${ }^{8}$ We introduce this parameter, because, with binary risk, the correlation coefficient of spouses' incomes can take three values only: $-1,0$, and 1 . The continuous $\varepsilon$ allows to distinguish further subcases which result in different matching predictions.
} 
$v$ to $\operatorname{Mr} j$, given $\beta$ and $\varepsilon$. Formally, we have

$$
\begin{aligned}
C_{i j}(v ; \beta, \varepsilon) & \equiv \arg \max _{C} \mathbb{E} u(i, C) \\
\text { s. t. } \quad & \mathbb{E} u(j, Z-C) \geq v, \\
& u(i, \bar{c})+\frac{\beta}{1-\beta} \mathbb{E} u(i, C) \geq u(i, 1+\varepsilon y)+\frac{\beta}{1-\beta} \mathbb{E} u(i, W), \\
& u(i, \underline{c})+\frac{\beta}{1-\beta} \mathbb{E} u(i, C) \geq u(i, 1-\varepsilon y)+\frac{\beta}{1-\beta} \mathbb{E} u(i, W), \\
& u(j, \bar{z}-\bar{c})+\frac{\beta}{1-\beta} \mathbb{E} u(j, Z-C) \geq u(j, 1+y)+\frac{\beta}{1-\beta} \mathbb{E} u(j, M), \\
& u(j, \underline{z}-\underline{c})+\frac{\beta}{1-\beta} \mathbb{E} u(j, Z-C) \geq u(j, 1-y)+\frac{\beta}{1-\beta} \mathbb{E} u(j, M) .
\end{aligned}
$$

The first constraint, (2), is the promise-keeping constraint (PKC). The remaining four constraints are the participation/enforcement constraints (PCs). These require that the contract be sustained voluntarily. That is, honouring the contract must be preferred to autarky, whatever the realised state. The right-hand sides capture the present value of autarky in the respective states. We say that a sharing rule is feasible if it satisfies all PCs (3)-(6).

Recall that negative sorting under full commitment (see Proposition 1) is driven by the comparative advantage of heterogeneous couples when sharing aggregate risk. Without constraints on transfers, diversity generates a higher surplus. In the following, we determine the qualities of an 'attractive partner' when transfers are constrained by limited commitment.

First, recall that an optimal sharing rule in which the PCs are slack must equate the between-state MRS of the two spouses. If the PCs are binding, however, agents may only be able to partially reduce the difference in MRS compared to autarky. We can therefore distinguish two cases:

1. $x(i, W) \geq x\left(i, C_{i j}(v ; \beta, \varepsilon)\right) \geq x\left(j, Z-C_{i j}(v ; \beta, \varepsilon)\right) \geq x(j, M)$,

2. $x(i, W) \leq x\left(i, C_{i j}(v ; \beta, \varepsilon)\right) \leq x\left(j, Z-C_{i j}(v ; \beta, \varepsilon)\right) \leq x(j, M)$.

In the first case, the man makes a positive net transfer in state $\bar{s}$, and the relevant PCs are (4) and (5). In the second case, he does so in state $\underline{s}$, and the relevant PCs are (3) and (6). ${ }^{9}$ Note that this distinction is fully determined by exogenous parameters. Appendix B describes the relevant PCs as a function of $\varepsilon$ and the spouses' risk aversion.

Clearly, any contract which is the outcome of (constrained-)efficient risk sharing must satisfy one of the two previous conditions. From now on, we restrict our attention to candidate contracts defined accordingly.

\footnotetext{
${ }^{9}$ If $x(i, W)=x(j, M)$, the autarky and the perfect-risk-sharing outcomes are the same, and no transfers are needed.
} 
Definition 4 (Candidate contracts). We say that a contract $C_{i j}(v ; \beta, \varepsilon)$ is a candidate if it is feasible and it satisfies $x\left(i, C_{i j}(v ; \beta, \varepsilon)\right) \geq x\left(j, Z-C_{i j}(v ; \beta, \varepsilon)\right)(\leq)$ given $x(i, W) \geq$ $x(j, M)(\leq)$.

Having determined the direction of transfers in each state, we wish to identify when risk aversion helps to commit to positive transfers. To do so, we first define a notion of advantage of a woman (man) over another.

Definition 5 (Advantage). We say that Ms $i$ has an advantage over $M s \tilde{i}$ when sharing risk with $M r j$, given $\beta$ and $\varepsilon$, if every candidate contract for the couple $\langle\tilde{i}, j\rangle$ satisfies the PCs of $M s i$. That is, if $C_{\tilde{i} j}(v ; \beta, \varepsilon)=\left(\bar{c}_{\tilde{i} j}(v ; \beta, \varepsilon), \underline{c}_{\tilde{i} j}(v ; \beta, \varepsilon)\right)$ is a candidate contract, then

$$
\begin{aligned}
& u\left(i, \bar{c}_{\tilde{i} j}(v ; \beta, \varepsilon)\right)+\frac{\beta}{1-\beta} \mathbb{E} u\left(i, C_{\tilde{i} j}(v ; \beta, \varepsilon)\right) \geq u(i, 1+\varepsilon y)+\frac{\beta}{1-\beta} \mathbb{E} u(i, W), \\
& u\left(i, \underline{c}_{\tilde{i} j}(v ; \beta, \varepsilon)\right)+\frac{\beta}{1-\beta} \mathbb{E} u\left(i, C_{\tilde{i} j}(v ; \beta, \varepsilon)\right) \geq u(i, 1-\varepsilon y)+\frac{\beta}{1-\beta} \mathbb{E} u(i, W) ;
\end{aligned}
$$

and similarly for men.

Given our definition, we are now ready to determine under which condition more riskaverse agents have an advantage, and when less risk-averse agents do.

Lemma 1. Take $\beta$ and $\varepsilon$ as given.

(i) If for all candidate contracts $C$ for the couple $\left\langle i^{\prime}, j\right\rangle$ the woman's consumption is less Jewitt-risky than her income, i.e., $C \succsim_{S C} W$, then $M s i^{\prime \prime}$ has an advantage;

(ii) If for all candidate contracts $C$ for the couple $\left\langle i^{\prime \prime}, j\right\rangle$ the woman's consumption is more Jewitt-risky than her income, i.e., $C \precsim_{S C} W$, then $M s i^{\prime}$ has an advantage; and similarly for men.

Proof. In Appendix A.

Intuitively, Lemma 1 provides conditions on the endogenous sharing rules which determine whether heterogenous or homogenous couples have a comparative advantage in enforcing risk sharing. In particular, if consumption of both men and women are less risky than their respective endowments, then more risk-averse agents have an advantage, and hence homogenous couples can sustain more risk sharing. On the other hand, if consumption is more risky than income on one side of the market but less risky on the other side, heterogenous couples have a comparative advantage.

Next we determine whether a reduction in risk on one side of the market increases the partner's risk. The following lemma provides sufficient conditions for two cases: if risk is reduced on the women's side, then risk must increase (decrease) on the men's side. 
Lemma 2. Let $C$ and $\widetilde{C}$ be candidate contracts, and assume that $\widetilde{C}$ is an increased spread of $C$.

(i) If $C, \widetilde{C} \in \mathbf{C}^{-}$, then the man's consumption $Z-C$ is a reduced spread of $Z-\widetilde{C}$;

(ii) If $C, \widetilde{C} \in \mathbf{C}^{+}$, then the man's consumption $Z-C$ is an increased spread of $Z-\widetilde{C}$.

Proof. In Appendix A.

Lemma 2 provides conditions under which we have a clear answer to the question which side of the market provides insurance to the other. Part (i) concerns situations in which there is scope for mutual insurance. If the commitment problem is severe, a couple may fail to insure idiosyncratic (zero-sum) risk sufficiently, and the woman may consume more in state $\underline{s}$; while men always consume more in state $\bar{s}$. In this case, a marginal increase in transfers reduces consumption risk on both sides. Instead, part (ii) concerns situations in which spouses' consumption processes are comonotone. In this case, consumption risk on the men's side can only be reduced at the expense of increased risk on the women's side, hence women provide insurance to men; or vice versa. ${ }^{10}$

Lemmas 1 and 2 are key to provide sufficient conditions for PAM and NAM with limited commitment. Combined, they also contain the main intuition for when types are compliments or substitutes. Intuitively, PAM requires (i) more risk-averse agents to have an advantage on both sides of the market, and (ii) that spouses can mutually reduce their consumption risk through transfers. Similarly, NAM requires (i) less risk-averse agents on one side and more risk-averse agents on the other side to have an advantage, and (ii) that a reduction of risk on one side increases risk on the other side of the market. Next we make these statements formal and provide sufficient conditions on the exogenous parameters of the model that guarantee them.

We first provide a sufficient condition for GDD, and in turn NAM. We seek restrictions on parameters which result in a comparative advantage for couples with more diverse risk attitudes. First, we need that partners cannot mutually reduce consumption risk through transfers. That is, we need that any candidate contract $C$ be more Jewitt-risky than the woman's endowment if and only if the man's contract $Z-C$ is less Jewitt-risky than his endowment. In this case, by Lemma 1, a less risk-averse woman and a more risk-averse man have comparative advantages. Second, we seek that competing candidate contracts $C$ and $\widetilde{C}$ be ranked by Jewitt's order as follows: $C \succsim_{S C} \widetilde{C}$ if and only if the opposite ranking holds on the men's side, i.e., $Z-\widetilde{C} \succsim_{S C} Z-C$. That is, among any two candidate contracts, the one

\footnotetext{
${ }^{10}$ Note that Lemma 2 does not extend to cases in which the contracts $C$ and $\widetilde{C}$ are not comonotone. In this case, $Z-C$ and $Z-\widetilde{C}$ cannot be ranked in general.
} 
that is less Jewitt-risky for women is more Jewitt-risky for men, and vice versa; case (ii) of Lemma 2. It turns out that both of these conditions are satisfied whenever $\varepsilon \geq 0$, hence we have the following result:

Proposition 2. If $\varepsilon \geq 0$, then the economy satisfies $N A M$.

Proof. In Appendix A.

Proposition 2 says that, as long as endowments covary positively, the NAM result under full commitment is robust to limited commitment. Note, however, that if the commitment problem is so severe that no couple can commit to positive transfers, the economy trivially satisfies both PAM and NAM according to Definition 2. This is because, in this case, each agent gets their autarky utility in any potential couple, hence any matching is stable according to Definition 1.

We now turn to showing that this is not true when endowments covary negatively. We first provide a sufficient condition for PAM in terms of equilibrium objects. This will be useful to determine sorting in terms of the exogenous parameters of our model below.

Lemma 3. If $C_{i j}(v ; \beta, \varepsilon) \in \mathbf{C}^{-}$for all $v$ and for all $\langle i, j\rangle$, then the economy satisfies PAM.

Proof. In Appendix A.

Lemma 3 says that PAM obtains whenever women's consumption is weakly higher in state $\underline{s}$ than in state $\bar{s}$. In other words, whenever spouses' consumptions violate comonotonicity, we have PAM. Note that this property is contrary to what happens under perfect risk sharing in the presence of aggregate risk.

Consider first the case where $\varepsilon=-1$, i.e., there is no aggregate risk and women's income is $1-y$ in state $\bar{s}$ and $1+y$ in state $\underline{s}$. In this case, even efficient transfers satisfy the sufficient condition for PAM of Lemma 3, since they result in equal consumptions in the two states. Therefore, we obtain the following result.

Proposition 3. If $\varepsilon=-1$, then the economy satisfies PAM.

Proof. In Appendix A.

Notice that PAM holds for any $\beta$ when the joint endowment is constant, whatever the level of risk sharing. The case of $\varepsilon=-1$ has the unique property that the partner's ability to share aggregate risk is irrelevant, even if the couple achieves efficient risk sharing. At the same time, a partner's risk aversion always increases the set of candidate contracts. As a result, more risk-averse agents are more attractive risk-sharing partners on both sides of 
the market to anyone on the opposite side. However, given that the joint endowment is constant, when $\beta$ is high enough to allow perfect risk sharing in all potential couples, we can generate a new payoff-equivalent stable matching by rearranging partners, hence both PAM and NAM hold. Similarly, if $\beta$ is so low that no potential couple achieves positive transfers, the economy satisfies both PAM and NAM (as in the case of $\varepsilon \geq 0$, see above).

With aggregate risk, i.e., $\varepsilon>-1$, all efficient risk-sharing contracts prescribe strictly higher consumption for both partners in $\bar{s}$. Therefore, the sufficient condition for PAM in Lemma 3 requires all couples to be constrained in equilibrium. Moreover, if incomes are comonotone, i.e., $\varepsilon \geq 0$, any constrained-efficient transfer must preserve comonotonicity of consumptions. As a result, the sufficient condition for PAM requires that idiosyncratic risk is great enough to satisfy $\varepsilon<0$.

In the following we determine the set of exogenous parameters, $\beta$ and $\varepsilon$, which give rise to our sufficient condition, $C_{i j}(v ; \beta, \varepsilon) \in \mathbf{C}^{-}$for all $v$ and for all $\langle i, j\rangle$, given $y$ and the current utility functions. Intuitively, we need the commitment problem to be severe such that consumptions fail to be comonotone.

In order to state our main result, let $\mathrm{Ms} I(\mathrm{Mr} J)$ be the most risk-averse woman (man).

Proposition 4. For any $\varepsilon<0$ there exists a discount factor $\bar{\beta}(\varepsilon) \in(0,1)$ such that the economy satisfies $P A M$ for all $\beta \leq \bar{\beta}(\varepsilon)$. Further, the threshold discount factor is implicitly given by

$$
C_{I J}(v ; \bar{\beta}(\varepsilon), \varepsilon) \in \mathbf{C}^{-} \text {for all } v \text { and } C_{I J}(v ; \bar{\beta}(\varepsilon), \varepsilon) \in \mathbf{C}^{-} \cap \mathbf{C}^{+} \text {for some } v \text {. }
$$

Proof. In Appendix A.

Proposition 4 says that the economy satisfies PAM when incomes covary negatively and the discount factor is sufficiently low. Contrary to the case where $\varepsilon=-1$, each couple faces aggregate risk, hence heterogeneity in risk preferences may be beneficial. Still, more riskaverse agents on both sides of the market have an advantage in sharing idiosyncratic risk. Our result shows that the latter can dominate when transfers are sufficiently constrained, which corresponds to lower discount factors, everything else equal.

The comparative statics with respect to $\varepsilon$ are not as clear-cut. While an increase in $\beta$ relaxes all PCs unambiguously, the comparative statics with respect to $\varepsilon$ are not monotone. Decreasing a negative $\varepsilon$ increases a woman's idiosyncratic income risk from an ex-ante perspective. However, depending on her risk preferences, this change may increase or decrease her autarky utility conditional on a high current income realisation (which is when her PC is binding). As a result, the PC may tighten or relax as $\varepsilon$ decreases. 
Nonetheless, we are able to identify situations in which both relevant PCs unambiguously tighten (or relax) as $\varepsilon$ changes. These allow us to determine the comparative statics of the commitment problem with respect to $\varepsilon$, and in turn to provide sufficient conditions for PAM. To state our result, let $\varepsilon_{I}(\beta)\left(\varepsilon_{J}(\beta)\right)$ denote the level of $\varepsilon$ where Ms $I$ enjoys the highest possible sure consumption such that her (Mr J's) PC holds as equality. ${ }^{11}$

Proposition 5. Assume that $C_{I J}\left(v ; \bar{\beta}\left(\varepsilon_{0}\right), \varepsilon_{0}\right) \in \mathbf{C}^{-}$for all $v$ holds for some $\varepsilon_{0} \in[-1,0)$.

(i) If $\varepsilon_{J}(\beta) \leq \varepsilon_{0} \leq \varepsilon_{I}(\beta)$, then the economy satisfies $P A M$ for all $\varepsilon \in\left[\varepsilon_{0}, \varepsilon_{I}(\beta)\right]$.

(ii) If $\varepsilon_{I}(\beta) \leq \varepsilon_{0} \leq \varepsilon_{J}(\beta)$, then the economy satisfies PAM for all $\varepsilon \in\left[\varepsilon_{J}(\beta), \varepsilon_{0}\right]$.

Proof. In Appendix A.

Below we show that non-monotonicity may arise by a numerical example as well, see Section 3.1.1.

To summarise, we have shown that for $\varepsilon=-1$, the economy satisfies PAM. Further, for any $-1<\varepsilon<0$, we have provided sufficient conditions for PAM in terms of the PCs of the two most risk-averse agents on each side of the market. If PCs of Ms $I$ and $\mathrm{Mr} J$ are tight enough for consumption not to be comonotone after transfers, then more risk-averse agents are more attractive partners. Moreover, we have shown that the sufficient condition holds when agents are impatient enough, i.e., $\beta$ is below a threshold $\bar{\beta}(\varepsilon)$, which depends on exogenous parameters only. However, the comparative statics of the PCs with respect to $\varepsilon$ are not monotone on the interval $(-1,0)$. In other words, $\bar{\beta}(\varepsilon)$ is not monotone in $\varepsilon$.

The intuition for our results in this section is as follows. When efficient transfers are not credible, a partner may become more attractive if he or she considers autarky a more severe punishment. The extent of the commitment problem is therefore unambiguously increasing (decreasing) in risk aversion if transfers make consumption more (less) Jewitt-risky than income. For $\varepsilon<0$ and low $\beta$, transfers reduce consumption risk on both sides of the market, while for $\varepsilon \geq 0$, transfers increase risk on one side while decreasing it on the other side. The clear sorting prediction in these cases exploits that the competing offers of two women for any given man can be ranked in terms of Jewitt's order. The sorting of couples for intermediate cases with $\varepsilon<0$ and high $\beta$ may be positive assortative if the sorting is driven by the couples' ability to pool idiosyncratic risk, negative if it is driven by the allocation of uninsurable risk, or non-monotone. ${ }^{12}$

\footnotetext{
${ }^{11}$ See formal definition in Appendix A.

${ }^{12}$ For instance, consider a group of men and women $i_{L}^{\prime}, i_{L}^{\prime \prime}, i_{H}^{\prime}, i_{H}^{\prime \prime}$ with $i_{L}^{\prime}<i_{L}^{\prime \prime}<i_{H}^{\prime}<i_{H}^{\prime \prime}$, and $j_{L}^{\prime}, j_{L}^{\prime \prime}, j_{H}^{\prime}, j_{H}^{\prime \prime}$ with $j_{L}^{\prime}<j_{L}^{\prime \prime}<j_{H}^{\prime}<j_{H}^{\prime \prime}$. Let $\beta$ be sufficiently high to allow for efficient risk sharing in any couple formed by Ms $i_{L}^{\prime}$ or $i_{L}^{\prime \prime}$ and $\mathrm{Mr} j_{H}^{\prime}$ or $j_{H}^{\prime \prime}$. Therefore, by the standard negative-sorting result for efficient risk sharing,
} 
We want to emphasise that our comparative statics results do not require the outside options to correspond to autarky or, more generally, to be exogenous. Consider, for instance, a situation in which the outside option is a convex combination between continuation values inside and outside the marriage. This accommodates a situation in which spouses withhold transfers for $0<k \leq \infty$ periods, or where a separated agent can remarry only after $k$ periods. That is, the man does not benefit from risk sharing for $k$ periods, and we assume that he can expect to get the same level of lifetime utility, $v$, thereafter. The man's constraint (5) would then become

$$
u(j, \bar{z}-\bar{c})+\frac{\beta-\beta^{k+1}}{1-\beta} \mathbb{E} u(j, Z-C) \geq u(j, \bar{m})+\frac{\beta-\beta^{k+1}}{1-\beta} \mathbb{E} u(j, M),
$$

where $\left(\beta-\beta^{k+1}\right) /(1-\beta)=\sum_{t=1}^{k} \beta^{t}$. Our benchmark case corresponds to $k=\infty$. A finite $k$ clearly affects the set of sharing rules which are self-enforcing. However, it does not affect the comparative statics with respect to risk aversion in our analysis. Moreover, we can accommodate a gender-specific $k$. Similarly, the outside options could be any gender-specific i.i.d. processes, $W^{\text {out }}$ for women and $M^{\text {out }}$ for men, for example additional punishment for deviating could be taken into account. In short, as long as the set of candidate sharing rules in any couple $\langle i, j\rangle$ can be compared to the outside option by Jewitt's single-crossing condition, our results apply.

\subsubsection{A numerical example}

To see how $\bar{\beta}(\varepsilon)$ changes with $\varepsilon$ and to illustrate the strength of our sufficient condition for PAM, we consider a set of parameterised economies and compute $\bar{\beta}(\varepsilon)$ for all $\varepsilon \in(-1,0)$. In addition, we provide the values of $\beta^{*}(\varepsilon)$, the threshold discount factor below which the couple $\langle I, J\rangle$ and hence all potential couples are in autarky, and $\beta^{* *}(\varepsilon)$, the threshold discount factor above which the least risk-averse couple and hence all potential couples achieve perfect risk sharing. ${ }^{13}$

For this purpose, we consider economies with two men and two women whose risk preferences satisfy constant relative risk aversion (CRRA), in particular, $u\left(i^{\prime}, c\right)=u\left(j^{\prime}, c\right)=$

the stable matching must include the couples $\left\langle i_{L}^{\prime}, j_{H}^{\prime \prime}\right\rangle$ and $\left\langle i_{L}^{\prime}, j_{H}^{\prime \prime}\right\rangle$. Now let $C_{w}=\left(c_{i_{H}^{\prime \prime}}(\beta, \varepsilon), c_{i_{H}^{\prime \prime}}(\beta, \varepsilon)\right)$. Then, for any $\beta$, we can construct a utility function for $\mathrm{Mr} j_{L}^{\prime \prime}$ such that $Z-C_{w}$ satisfies his $\mathrm{PC}$ as equality. Therefore, all candidate contracts for the couple $\left\langle i_{H}^{\prime \prime}, j_{L}^{\prime \prime}\right\rangle$ must belong to $\mathbf{C}^{-}$. Moreover, the $\mathrm{PC}$ of $\mathrm{Mr} j_{L}^{\prime}$ is more stringent than the one of $\mathrm{Mr} j_{L}^{\prime \prime}$. Similarly, the $\mathrm{PC}$ of $\mathrm{Ms} i_{H}^{\prime}$ is more stringent than the one of Ms $i_{H}^{\prime \prime}$ for all candidate contracts with $\mathrm{Mr} j_{L}^{\prime}$ and $\mathrm{Mr} j_{L}^{\prime \prime}$. As a result, no stable matching includes the couples $\left\langle i_{H}^{\prime \prime}, j_{L}^{\prime}\right\rangle$ and $\left\langle i_{H}^{\prime}, j_{L}^{\prime \prime}\right\rangle$ at the same time. By the above, there exists a profile of utility promises such that the only stable matching is $\left\{\left\langle i_{H}^{\prime} j_{L}^{\prime}\right\rangle,\left\langle i_{H}^{\prime \prime} j_{L}^{\prime \prime}\right\rangle,\left\langle i_{L}^{\prime} j_{H}^{\prime \prime}\right\rangle,\left\langle i_{L}^{\prime \prime} j_{H}^{\prime}\right\rangle\right\}$. To summarise, $i_{H}^{\prime \prime} \geq i_{H}^{\prime} \geq i_{L}^{\prime \prime}$ while $\mu\left(i_{H}^{\prime \prime}\right)=j_{L}^{\prime \prime} \geq \mu\left(i_{H}^{\prime}\right)=j_{L}^{\prime} \leq \mu\left(i_{L}^{\prime \prime}\right)=j_{H}^{\prime \prime}$, hence monotonicity is violated.

${ }^{13}$ Ligon, Thomas, and Worrall $(2002)$ show that $\beta^{*}(\varepsilon)$ exists. $\beta^{* *}(\varepsilon)$ exists by a standard folk-theorem result, see Kimball (1988) and Kocherlakota (1996). 
$c^{1-1.5} /(1-1.5)=-2 / \sqrt{c}$ and $u\left(i^{\prime \prime}, c\right)=u\left(j^{\prime \prime}, c\right)=c^{1-2} /(1-2)=-1 / c$. The coefficient of relative risk aversion is therefore 1.5 for $\mathrm{Ms} i^{\prime}$ and $\mathrm{Mr} j^{\prime}$ and 2 for Ms $i^{\prime \prime}$ and $\mathrm{Mr} j^{\prime \prime}$. Further, we set $y=0.1$, hence men's income is $M=(1.1,0.9)$.

Figure 1 plots $\bar{\beta}(\varepsilon), \beta^{*}(\varepsilon)$, and $\beta^{* *}(\varepsilon)$ for all $\varepsilon \in(-1,0)$. Given $\varepsilon$, our sufficient condition for $\mathrm{PAM}$ is satisfied for all $\beta \leq \bar{\beta}(\varepsilon)$. Pairs of parameters $\varepsilon$ and $\beta$ in the shaded area correspond to cases where PAM holds and at least the most risk-averse couple shares risk partially. ${ }^{14}$ When all potential couples are in autarky, both PAM and NAM hold. When all couples share risk efficiently, NAM holds for all $\varepsilon$, and PAM holds in addition to NAM in the special case of $\varepsilon=-1$. Finally, when $\varepsilon=-1$ and $\beta$ is such that some couples achieve some but less than perfect risk sharing, we know that PAM holds.

Figure 1: PAM with static contracts

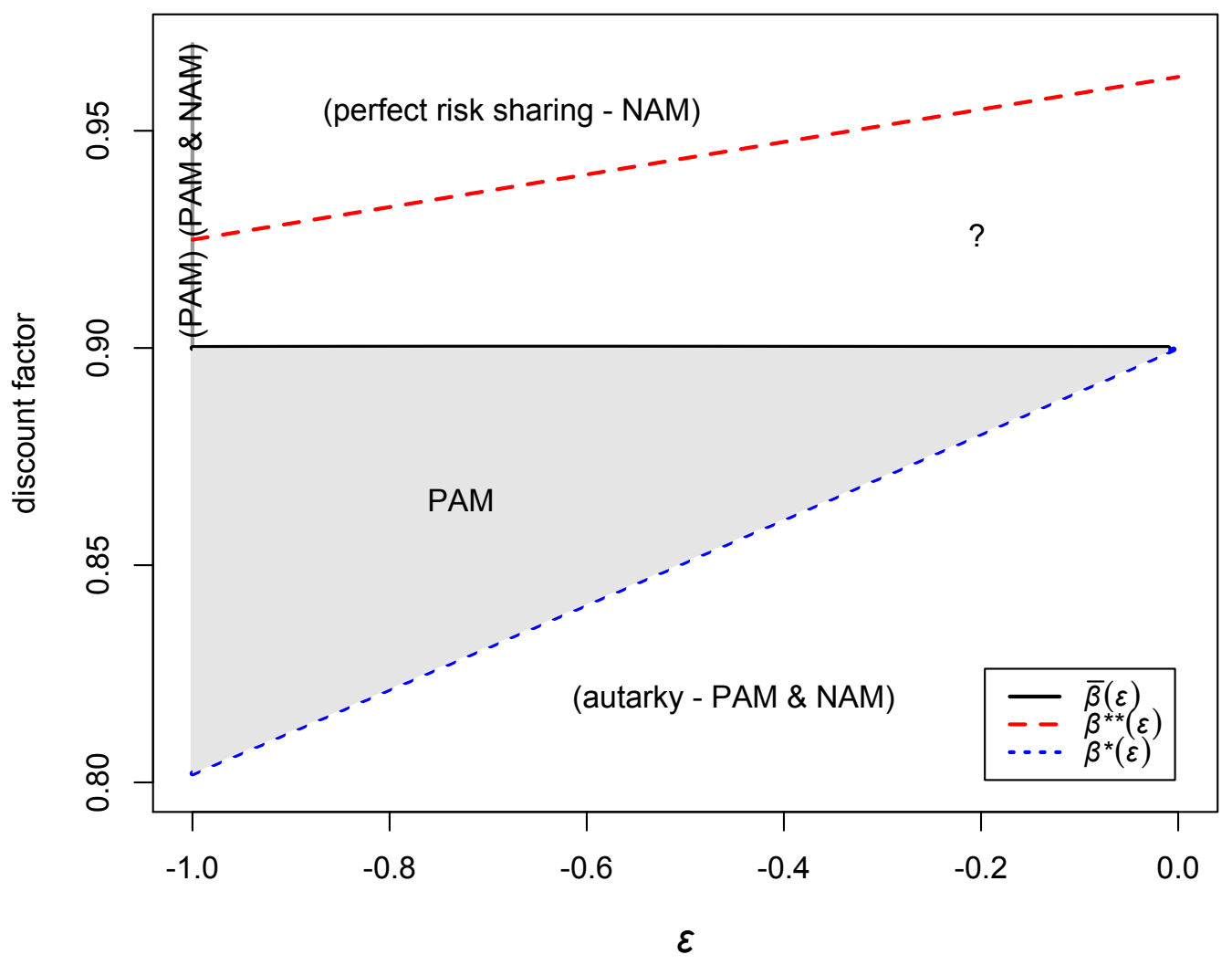

Notes: $\bar{\beta}(\varepsilon)$ is the discount factor below which $C_{i^{\prime \prime} j^{\prime \prime}}(v ; \beta, \varepsilon) \in \mathbf{C}^{-}$for all $v$, a sufficient condition for PAM. $\beta^{*}(\varepsilon)$ is the discount factor below which no transfers are self-enforcing for the couple $\left\langle i^{\prime \prime}, j^{\prime \prime}\right\rangle . \beta^{* *}(\varepsilon)$ is the discount factor above which perfect risk sharing is self-enforcing in the long run for the couple $\left\langle i^{\prime}, j^{\prime}\right\rangle$. The shaded area highlights the combinations of $\varepsilon$ and $\beta$ for which our sufficient condition for PAM holds when $\varepsilon \in[-1,0)$ and at least the couple $\left\langle i^{\prime \prime}, j^{\prime \prime}\right\rangle$ achieves partial risk sharing.

\footnotetext{
${ }^{14}$ While our sufficient conditions for PAM do not imply the absence of NAM, we have not been able to find a stable matching that is negative assortative for $\beta \in\left(\beta^{*}(\varepsilon), \bar{\beta}(\varepsilon)\right]$.
} 
In Figure 2 we plot $\bar{\beta}(\varepsilon)$ separately as a function of $\varepsilon$. Its hump shape illustrates that, for some values of $\beta$, PAM may not hold if $\varepsilon$ is too close to either 0 or -1 . For example when $\beta=0.90005$, our sufficient condition is satisfied for all $\varepsilon \in[-0.87,-0.42]$.

Figure 2: PAM with static contracts - non-monotonicity of $\bar{\beta}(\varepsilon)$

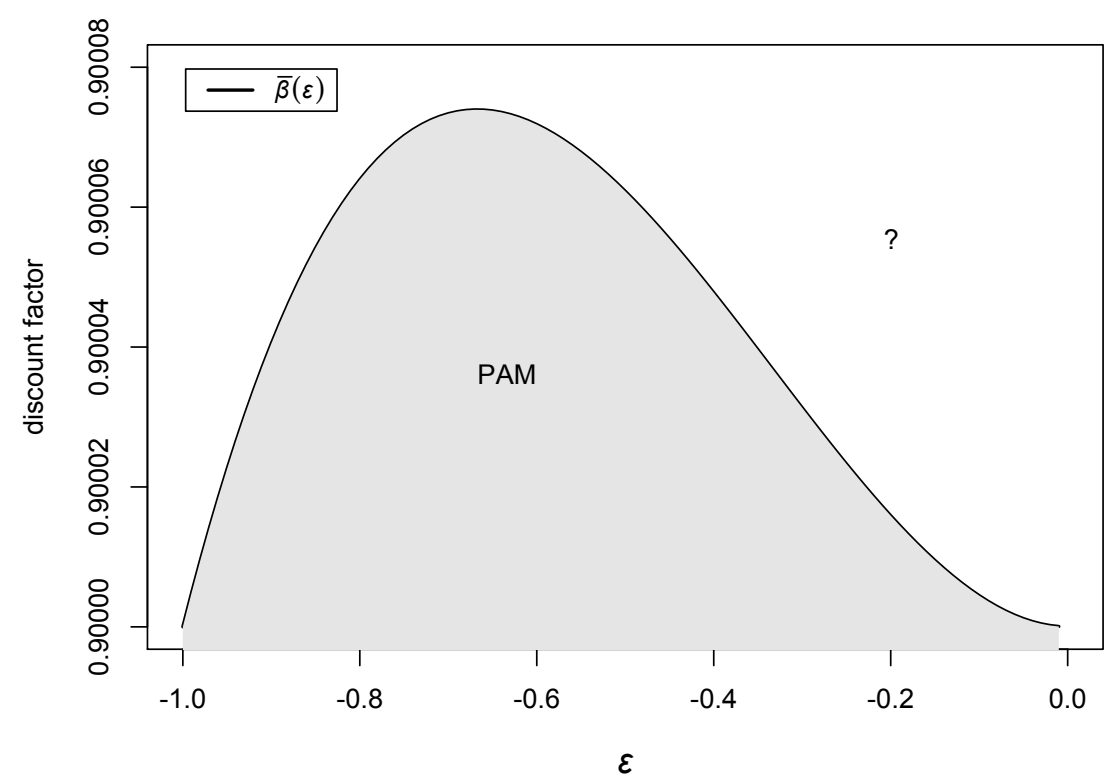

Notes: $\bar{\beta}(\varepsilon)$ is the discount factor below which $C_{i^{\prime \prime} j^{\prime \prime}}(v ; \beta, \varepsilon) \in \mathbf{C}^{-}$for all $v$, a sufficient condition for PAM.

\subsection{Dynamic contracts}

The static contracts discussed in the previous section are not constrained efficient in a dynamic risk-sharing model with limited commitment (Thomas and Worrall, 1988; Kocherlakota, 1996). That is, Ms $i$ could provide the expected per-period utility $v$ to $\operatorname{Mr} j$ and increase her own utility by proposing a self-enforcing dynamic contract, which conditions on the entire history of income realisations. To allow for history-depending contracts when determining sorting, we need to turn to numerical methods. The aim of this section is to demonstrate that the existence of positive-assortative matchings is robust to constrainedefficient risk sharing.

To characterise dynamic contracts, we need additional notation. Let $s_{t} \in\{\bar{s}, \underline{s}\}$ denote the state of the world at time $t$, let $s^{t}=\left(s_{1}, s_{2}, \ldots, s_{t}\right)$ denote the history of state realisations up to and including time $t$, and let $\operatorname{Pr}\left(s^{t}\right)$ denote its probability. Further, let $c\left(s^{t}\right)\left(z\left(s_{t}\right)-c\left(s^{t}\right)\right)$ denote consumption by the woman (the man) at time $t$ when history $s^{t}$ has occurred. The optimal contract solves 


$$
\begin{aligned}
& \max _{\left\{c\left(s^{t}\right)\right\}} \sum_{t=1}^{\infty} \sum_{s^{t}} \operatorname{Pr}\left(s^{t}\right) \beta^{t-1} u\left(i, c\left(s^{t}\right)\right) \\
& \text { s. t. } \quad \sum_{t=1}^{\infty} \sum_{s^{t}} \operatorname{Pr}\left(s^{t}\right) \beta^{t-1} u\left(j, c\left(s^{t}\right)\right) \geq \frac{1}{1-\beta} v, \\
& u\left(i, c\left(s^{t-1}, \bar{s}\right)\right)+\sum_{r=t+1}^{\infty} \sum_{s^{r} \supset s^{t}} \operatorname{Pr}\left(s^{r} \mid s^{t}\right) \beta^{r-t} u\left(i, c\left(s^{r}\right)\right) \geq u(i, \bar{w})+\frac{\beta}{1-\beta} \mathbb{E} u(i, W), \forall s^{t}, \\
& u\left(i, c\left(s^{t-1}, \underline{s}\right)\right)+\sum_{r=t+1}^{\infty} \sum_{s^{r} \supset s^{t}} \operatorname{Pr}\left(s^{r} \mid s^{t}\right) \beta^{r-t} u\left(i, c\left(s^{r}\right)\right) \geq u(i, \underline{w})+\frac{\beta}{1-\beta} \mathbb{E} u(i, W), \forall s^{t}, \\
& u\left(j, z(\bar{s})-c\left(s^{t-1}, \bar{s}\right)\right)+\sum_{r=t+1}^{\infty} \sum_{s^{r} \supset s^{t}} \operatorname{Pr}\left(s^{r} \mid s^{t}\right) \beta^{r-t} u\left(j, z\left(s_{r}\right)-c\left(s^{r}\right)\right) \geq u(j, \bar{m})+\frac{\beta}{1-\beta} \mathbb{E} u(j, M), \forall s^{t}, \\
& u\left(j, z(\underline{s})-c\left(s^{t-1}, \underline{s}\right)\right)+\sum_{r=t+1}^{\infty} \sum_{s^{r} \supset s^{t}} \operatorname{Pr}\left(s^{r} \mid s^{t}\right) \beta^{r-t} u\left(j, z\left(s_{r}\right)-c\left(s^{r}\right)\right) \geq u(j, \underline{m})+\frac{\beta}{1-\beta} \mathbb{E} u(j, M), \forall s^{t},
\end{aligned}
$$

where $\operatorname{Pr}\left(s^{r} \mid s^{t}\right)$ is the probability of history $s^{r} \supset s^{t}$ occurring given that history $s^{t}$ occurred up to time $t$.

The characterisation of constrained-efficient allocations in endowment economies with limited commitment has been established in the literature, see Kocherlakota (1996), Ligon, Thomas, and Worrall (2002), and others. While this literature typically focuses on the properties of the long-run allocations, we are interested in matching at time 0 . That is, we need to take into account the transition to the long-run equilibrium, which allows promises of expected per-period utility above or below the long-run utility. During the possible transition period, no $\mathrm{PC}$ is binding and the sharing rule is determined by the utility promise to the man. As a result, we may need to determine a third level of consumption which may occur during the transition period, in addition to the long-run consumptions in states $\bar{s}$ and $\underline{s}$. Let $\hat{c}$ denote the woman's consumption during the possible transition period, i.e., before any PC binds. ${ }^{15}$

Let $\beta^{*}$ denote the discount factor below which autarky is the only feasible allocation and let $\beta^{* *}$ denote the discount factor above which efficient risk sharing is self-enforcing in the long run, as before. Then, for given preferences and income processes, the following cases may occur for a couple $\langle i, j\rangle$ depending on $\beta$ and $v$ :

(i) $\beta \leq \beta^{*}$. There is no transition. Consumption only takes two values, which are equal to the endowments, and the solution is independent of $v$.

(ii) $\beta^{*}<\beta<\beta^{* *}$. Both relevant PCs bind in the long run, and they pin down the long-run

\footnotetext{
${ }^{15}$ Note that the transition period is finite with probability one. For instance, if the state space is binary, then period $t$ features the long-run sharing rule if both states $\underline{s}$ and $\bar{s}$ occurred prior to $t$.
} 
consumption values, which are independent of $v \cdot{ }^{16}$ If the promised utility equals the expected long-run per-period utility, $v^{\mathrm{LR}}$, then there is no transition, a $\mathrm{PC}$ binds already at time 1 , and consumption is determined by the two long-run values. Otherwise, there may be a transition period in which no $\mathrm{PC}$ binds. For $v<v^{\mathrm{LR}}\left(v>v^{\mathrm{LR}}\right), \hat{c}$ is larger (smaller) than the long-run consumption values, and it is realised if the man happens to have low (high) income initially. The maximum (minimum) sustainable $\hat{c}$, and therefore the range of utility promises, is pinned down by the relevant $\mathrm{PC}$ of the man (woman) in the transition state. ${ }^{17}$

(iii) $\beta^{* *} \leq \beta$. Efficient risk sharing occurs in the long run and $v$ matters. For intermediate values of $v$, no $\mathrm{PC}$ ever binds, hence there is no transition, and two consumption values occur overall. For low and high values of $v$, one PC eventually binds in one period. If this happens at $t \geq 2$, there is a transition period, and three consumption values occur overall. The long-run consumption values are pinned down by the woman's relevant PC if $v$ is high and by the man's relevant $\mathrm{PC}$ if $v$ is low, along with the efficiency condition that the ratios of marginal utilities across states of the two spouses are equal. ${ }^{18}$

Whenever the transition consumption values can differ from their long-run levels, the contract is dynamic. That is, we need to take a novel effect into account when analysing the assortativity of stable matchings: whether risk aversion enhances or constrains an agent's ability to transfer utility during the transition. Beyond the added difficulties due to the dynamic nature of the contract, an analytical approach is further complicated by the fact that competing contracts need not satisfy Jewitt's single-crossing condition when consumption takes three values or more. Therefore, we turn to numerical methods to check whether our results extend to the general model allowing for history-dependent contracts.

To show that stable matchings can be positive assortative with dynamic contracts as well, we revisit our numerical example. Recall that $y=0.1$ and $u\left(i^{\prime}, c\right)=u\left(j^{\prime}, c\right)=c^{1-1.5} /(1-$ $1.5)=-2 / \sqrt{c}$ and $u\left(i^{\prime \prime}, c\right)=u\left(j^{\prime \prime}, c\right)=c^{1-2} /(1-2)=-1 / c$. We set $\varepsilon=-0.1$ and $\beta=0.9$. Given these parameter values, the couples $\left\langle i^{\prime}, j^{\prime}\right\rangle$ and $\left\langle i^{\prime \prime}, j^{\prime}\right\rangle$ cannot sustain positive transfers, hence the expected lifetime utility of $\mathrm{Mr} j^{\prime}$ is equal to the value of autarky. However, when in the couple $\left\langle i^{\prime}, j^{\prime \prime}\right\rangle$, Ms $i^{\prime}$ enjoys a long-run consumption of 0.99935 and 1.00222 in states $\bar{s}$ and $\underline{s}$, respectively. Making $\mathrm{Ms} i^{\prime}$ indifferent between her two potential partners requires a transition consumption of 0.99 in state $\bar{s}$. This contract gives $\mathrm{Mr} j^{\prime \prime}$ an expected per-period

\footnotetext{
${ }^{16}$ See Proposition 4.2 in Kocherlakota (1996).

${ }^{17}$ These statements follow form the updating rule for the relative Pareto weight as shown in Proposition 1 of Ligon, Thomas, and Worrall (2002).

${ }^{18}$ Proposition 4.1 and related discussion in Kocherlakota (1996) establish this characterisation.
} 
utility of -1.008526 . Finally, for the couple $\left\langle i^{\prime \prime}, j^{\prime \prime}\right\rangle$, the long-run consumption values of the woman are 1.000896 and 1.000905, respectively. A transition consumption of the woman of 0.9935 in state $\bar{s}$ makes $\mathrm{Mr} j^{\prime \prime}$ indifferent between his two potential partners, and makes Ms $i^{\prime \prime}$ better off than when she shares risk with $\mathrm{Mr} j^{\prime}$. Hence, we have found a stable matching that is positive assortative.

The intuition for the fact that allowing for a transition does not overturn the assortativity of the stable matching in our example is that more risk-averse agents are still able to commit to more risk sharing. To see this, denote the contract which satisfies both relevant PCs as equality in the long run by $C_{i j}^{L R}(\beta, \varepsilon)$. Our sufficient condition for PAM of Lemma 3 is equivalent to the property $C_{i j}^{L R}(\beta, \varepsilon) \in \mathbf{C}^{-}$. Moreover, consumption in the transition period lies between $[1+\varepsilon y, 1-\varepsilon y]$, otherwise a PC would be violated. This implies that the dynamic contract is less Jewitt-risky than the endowment, hence more risk-averse agents still have an advantage on both sides of the matching market.

\section{Concluding remarks}

Recent theoretical literature on the marriage problem (two-sided, one-to-one matching) in endowment economies with imperfectly transferable utility and no search frictions predicts that matching is always negative assortative with respect to spouses' risk attitudes. In this paper we have shown that this finding is not robust to relaxing the assumption of efficient risk sharing once matches are formed. When commitment is limited, positive-assortative matching can occur. ${ }^{19}$

The reason for positive assortativity is that more risk-averse agents can be more attractive risk-sharing partners when their relevant PC is less stringent, which happens when their consumption is less risky than their income in the sense of Jewitt. When incomes are negatively correlated and the discount factor is sufficiently low, this effect dominates the gains from diversity in risk preferences when sharing uninsurable risk.

Our result of positive-assortative matching on risk attitudes is in line with recent empirical evidence by Bacon, Conte, and Moffatt (2014) on married couples, who use the German Socio-Economic Panel (SOEP), which contains a measure of risk preferences. The authors' preferred estimate of the correlation coefficient between spouses' risk aversion is 0.285. Fur-

\footnotetext{
${ }^{19}$ In a similar vein, Ghatak and Karaivanov (2014) find that frictions on both sides of a market can reverse sorting from the first-best case. They consider the effect of double-sided moral hazard among risk-neutral landlords and tenants who produce output jointly and are heterogenous in their specialisation type. They show that while heterogeneous pairs maximise first-best surplus, this effect may be dominated by the reduced friction in homogeneous couples.
} 
ther, Shore (2015) estimates that spouses' incomes remain negatively correlated for at least 20 years, using the Panel Study of Income Dynamics (PSID). In addition, he finds positiveassortative matching with respect to volatility of incomes, and argues that this may be optimal given positive-assortative matching in risk aversion. While our model assumes that income processes are homogeneous and exogenous on each side of the market, our results may help resolve the tension between theories in which agents match to take risk, which predict homogeneous couples, and those where agents match to share risk, which predicted heterogeneous couples. Our paper suggests that risk sharing may also favour homogeneous couples.

Considering a second application, firms and workers, Falco (2014) studies the role of risk aversion in the allocation of workers between formal and informal jobs in Ghana, and finds that more risk-averse workers are less likely to have jobs in the informal sector, where income uncertainty is higher. Arguably, informal firms are smaller and behave in a more risk-averse fashion, hence positive-assortative matching obtains.

Our mechanism that lack of commitment provides a motive for positive-assortative matching could be tested using an exogenous change in enforcement technology. The widespread adoption of unilateral divorce laws in the United States in the 1970s could be looked at from this perspective. Employment laws that make it more likely that an entrepreneur and a worker separate would also be a force toward positive-assortative matching according to our theory. We leave this to future work. 


\section{References}

Abreu, D. (1988). On the Theory of Infinitely Repeated Games with Discounting. Econometrica 56(2), 383-396.

Anderson, A. and L. Smith (2010). Dynamic Matching and Evolving Reputations. Review of Economic Studies 7r(1), 3-29.

Bacon, P. M., A. Conte, and P. G. Moffatt (2014). Assortative Mating on Risk Attitude. Theory and Decision $77(3), 389-401$.

Borch, K. (1962). Equilibrium in a Reinsurance Market. Econometrica 30(3), 424-444.

Chiappori, P.-A. and P. J. Reny (2016). Matching to Share Risk. Theoretical Economics $11(1), 227-251$.

Coate, S. and M. Ravallion (1993). Reciprocity without Commitment: Characterization and Performance of Informal Insurance Arrangements. Journal of Development Economics 40, $1-24$.

Dercon, S. and P. Krishnan (2000). In Sickness and in Health: Risk Sharing within Households in Rural Ethiopia. Journal of Political Economy 108(4), 688-727.

Falco, P. (2014). Does Risk Matter for Occupational Choices? Experimental Evidence from an African Labour Market. Labour Economics 28(C), 96-109.

Ghatak, M. and A. Karaivanov (2014). Contractual Structure in Agriculture with Endogenous Matching. Journal of Development Economics 110, 239-249.

Gollier, C. (2001). The Economics of Risk and Time. MIT Press.

Guiso, L., L. Pistaferri, and F. Schivardi (2005). Insurance within the Firm. Journal of Political Economy 113(5), 1054-1087.

Hess, G. D. (2004, April). Marriage and Consumption Insurance: What's Love Got to Do with It? Journal of Political Economy 112(2), 290-318.

Jewitt, I. (1986). A Note on Comparative Statics and Stochastic Dominance. Journal of Mathematical Economics 15(3), 249-254.

Kimball, M. S. (1988). Farmers' Cooperatives as Behavior toward Risk. American Economic Review 78(1), 224-232. 
Kocherlakota, N. (1996). Implications of Efficient Risk Sharing without Commitment. Review of Economic Studies 63(4), 595-609.

Kotlikoff, L. J. and A. Spivak (1981, April). The Family as an Incomplete Annuities Market. Journal of Political Economy 89(2), 372-391.

Legros, P. and A. F. Newman (2007). Beauty Is a Beast, Frog Is a Prince: Assortative Matching with Nontransferabilities. Econometrica 75(4), 1073-1102.

Li, S., H. Sun, and P. Chen (2013). Assortative Matching of Risk-Averse Agents with Endogenous Risk. Journal of Economics 109(1), 27-40.

Ligon, E., J. P. Thomas, and T. Worrall (2002). Informal Insurance Arrangements with Limited Commitment: Theory and Evidence from Village Economies. Review of Economic Studies 69(1), 209-244.

Mazzocco, M. (2004). Saving, Risk Sharing, and Preferences for Risk. American Economic Review 94(4), 1169-1182.

Mazzocco, M. (2007). Household Intertemporal Behaviour: A Collective Characterization and a Test of Commitment. Review of Economic Studies 74(3), 857-895.

Ogaki, M. and Q. Zhang (2001). Decreasing Relative Risk Aversion and Tests of Risk Sharing. Econometrica 69(2), 515-526.

Rosenzweig, M. R. and O. Stark (1989, August). Consumption Smoothing, Migration, and Marriage: Evidence from Rural India. Journal of Political Economy 97(4), 905-926.

Rudanko, L. (2009). Labor Market Dynamics under Long-Term Wage Contracting. Journal of Monetary Economics 56(2), 170-183.

Sandmo, A. (1971). On the Theory of the Competitive Firm under Price Uncertainty. American Economic Review 61(1), 65-73.

Schulhofer-Wohl, S. (2006). Negative Assortative Matching of Risk-Averse Agents with Transferable Expected Utility. Economics Letters 92(3), 383-388.

Serfes, K. (2005). Risk Sharing vs. Incentives: Contract Design under Two-Sided Heterogeneity. Economics Letters 88(3), 343-349.

Shore, S. H. (2015). The Co-Movement of Couples? Incomes. Review of Economics of the Household 13(3), 569-588. 
Thomas, J. and T. Worrall (1988). Self-Enforcing Wage Contracts. Review of Economic Studies 55(4), 541-554.

Wang, X. Y. (2015). Risk Sorting, Portfolio Choice, and Endogenous Informal Insurance. NBER Working Paper no. 20429. 


\section{Appendices}

\section{A Proofs}

Proof of Proposition 1. Efficient risk sharing requires consumption to lie in $\mathbf{C}^{+}$for both risk sharing partners, Ms $i$ and $\operatorname{Mr} j$. Define the crossing point $X(j, v) \in \mathbf{C}^{+}$for each $(j, v)$ pair as $C^{*}\left(i^{\prime}, j, v\right) \sim_{i^{\prime}} X(j, v) \sim_{i^{\prime \prime}} C^{*}\left(i^{\prime \prime}, j, v\right)$. By revealed preference, $C^{*}\left(i^{\prime}, j, v\right) \succsim_{i^{\prime}}$ $X(j, v) \succsim_{i^{\prime}} C^{*}\left(i^{\prime \prime}, j, v\right)$ and $C^{*}\left(i^{\prime \prime}, j, v\right) \succsim_{i^{\prime \prime}} X(j, v) \succsim_{i^{\prime \prime}} C^{*}\left(i^{\prime}, j, v\right)$. Hence,

$$
C^{*}\left(i^{\prime \prime}, j, v\right) \succsim_{S C} X(j, v) \succsim_{S C} C^{*}\left(i^{\prime}, j, v\right), \forall(j, v)
$$

since Ms $i^{\prime \prime}$ is more risk averse than Ms $i^{\prime}$ by assumption. Suppose the premise of (GDD), i.e., Ms $i^{\prime \prime}$ prefers to share risk with Mr $j^{\prime \prime}$ rather than Mr $j^{\prime}$. By revealed preference over partners, $\mathbb{E} u\left(j^{\prime}, Z-C_{i^{\prime \prime} j^{\prime \prime}}^{*}\left(v^{\prime \prime}\right)\right) \leq v^{\prime}$. Otherwise, if $\mathrm{Mr} j^{\prime \prime}$ s utility constraint could be satisfied with the offer made to Mr $j^{\prime \prime}$, then Mr $j^{\prime \prime}$ would not be a preferred partner. Suppose first that Mr $j^{\prime \prime}$ agrees with $\mathrm{Mr} j^{\prime}$ on the ranking of the two offers, i.e., $\mathbb{E} u\left(j^{\prime \prime}, Z-C_{i^{\prime \prime} j^{\prime}}^{*}\left(v^{\prime}\right)\right) \geq v^{\prime \prime}$. Then $(7)$ together with $(Z-C) \in \mathbf{C}^{+}$implies $Z-C_{i^{\prime} j^{\prime}}^{*}\left(v^{\prime}\right) \succsim_{S C} Z-C_{i^{\prime \prime} j^{\prime}}^{*}\left(v^{\prime}\right)$. Therefore, $Z-C_{i^{\prime} j^{\prime}}^{*}\left(v^{\prime}\right) \succsim_{j^{\prime \prime}}$ $Z-C_{i^{\prime \prime} j^{\prime}}^{*}\left(v^{\prime}\right) \succsim_{j^{\prime \prime}} Z-C_{i^{\prime \prime} j^{\prime \prime}}^{*}\left(v^{\prime \prime}\right)$ and $\mathbb{E} u\left(j^{\prime \prime}, Z-C_{i^{\prime} j^{\prime}}^{*}\left(v^{\prime}\right)\right) \geq v^{\prime \prime}$. A standard separating hyperplane argument implies that Ms $i^{\prime}$ can find a $C \succsim_{i^{\prime}} C_{i^{\prime} j^{\prime}}^{*}\left(v^{\prime}\right)$ which provides exactly $v^{\prime \prime}$ to $\operatorname{Mr} j^{\prime \prime}$. That is, Ms $i^{\prime}$ can always outbid Ms $i^{\prime \prime}$ and match with Mr $j^{\prime \prime}$. Suppose now that Mr $j^{\prime \prime}$ disagrees with Mr $j^{\prime}$ on the ranking of the two offers, i.e., now $Z-C_{i^{\prime \prime} j^{\prime \prime}}^{*}\left(v^{\prime \prime}\right) \succsim_{j^{\prime \prime}} Z-C_{i^{\prime \prime} j^{\prime}}^{*}\left(v^{\prime}\right)$. Then $\exists \widetilde{C} \in \mathbf{C}^{+}$such that $\mathbb{E} u\left(j^{\prime}, Z-\widetilde{C}\right)=v^{\prime}$ and $\mathbb{E} u\left(j^{\prime \prime}, Z-\widetilde{C}\right)=v^{\prime \prime}$. The disagreement implies

$$
C_{i^{\prime \prime} j^{\prime}}^{*}\left(v^{\prime}\right) \succsim_{S C} \widetilde{C} \succsim_{S C} C_{i^{\prime \prime} j^{\prime \prime}}^{*}\left(v^{\prime \prime}\right)
$$

If $C_{i^{\prime} j^{\prime}}^{*}\left(v^{\prime}\right) \succsim_{S C} \widetilde{C}$, then we know that $X\left(j^{\prime}, v^{\prime}\right) \succsim_{S C} \widetilde{C} \succsim_{S C} X\left(j^{\prime \prime}, v^{\prime \prime}\right)$. Then, given that the more risk-averse agent $\mathrm{Ms} i^{\prime \prime}$ prefers to match with $\mathrm{Mr} j^{\prime \prime}$ by assumption, i.e., she prefers the more risky lottery $X\left(j^{\prime \prime}, v^{\prime \prime}\right)$ over the less risky lottery $X\left(j^{\prime}, v^{\prime}\right)$, it must be that the less risk-averse agent Ms $i^{\prime}$ also prefers $X\left(j^{\prime \prime}, v^{\prime \prime}\right)$ over $X\left(j^{\prime}, v^{\prime}\right)$. Further, (7) implies that $Z-C_{i^{\prime} j^{\prime \prime}}^{*}\left(v^{\prime \prime}\right) \succsim_{j^{\prime \prime}} Z-C_{i^{\prime \prime} j^{\prime \prime}}^{*}\left(v^{\prime \prime}\right)$. If $\widetilde{C} \succsim_{S C} C_{i^{\prime} j^{\prime}}^{*}\left(v^{\prime}\right)$, then $Z-C_{i^{\prime} j^{\prime}}^{*}\left(v^{\prime}\right) \succsim_{j^{\prime \prime}} Z-\widetilde{C} \sim_{j^{\prime \prime}}$ $Z-C_{i^{\prime \prime} j^{\prime \prime}}^{*}\left(v^{\prime \prime}\right)$, and again there exists a contract $C$ which is preferred by Ms $i^{\prime}$ and grants Mr $j^{\prime \prime}$ exactly $v^{\prime \prime}$, hence NAM.

Proof of Lemma 1. Assume first that the relevant PC is (3). Multiplying both sides of the inequality (3) by $(1-\beta)$, we can express (3) in terms of a static expected utility criterion which compares $C$ and $W$ as

$$
[(1-\beta)+\beta \operatorname{Pr}(\bar{s})] u(i, \bar{c})+\beta \operatorname{Pr}(\underline{s}) u(i, \underline{c}) \geq[(1-\beta)+\beta \operatorname{Pr}(\bar{s})] u(i, \bar{w})+\beta \operatorname{Pr}(\underline{s}) u(i, \underline{w}) \text {. }
$$


Consider first part (i), where $C$ is assumed less Jewitt-risky than $W$. By the definition of Jewitt's order, whenever the above inequality holds for Ms $i^{\prime}$, then it must also hold for Ms $i^{\prime \prime}$, which proves (i). Assume now that the relevant PC is (4). Multiplying both sides of the inequality in $(4)$ by $(1-\beta)$ yields a similar expected utility criterion which assigns weight $[(1-\beta)+\beta \operatorname{Pr}(\underline{s})]$ to state $\underline{s}$ and $\beta \operatorname{Pr}(\bar{s})$ to state $\bar{s}$. According to Jewitt's order, whenever $C \succsim_{S C} W$ satisfies the inequality for $\mathrm{Ms} i^{\prime}$ it must also satisfy the inequality for Ms $i^{\prime \prime}$, which proves (i). Applying an analogous argument to the cases where $W \succsim_{S C} C$ proves (ii). To show the results for men, we can apply a similar argument using the inequalities (5) and (6) and the riskiness of payoffs $Z-C$ and $M$.

Proof of Lemma 2. First, we show that any candidate contract $C$ must satisfy $Z-C \in \mathbf{C}^{+}$. Since $\bar{m} \geq \underline{m}$, we have that the man's individual income $M$ is comonotone with aggregate income $Z$. Moreover, since $\bar{z} \geq \underline{z}$, the inequality $\bar{z}-\bar{c} \leq \underline{z}-\underline{c}$ can only hold if women make a transfer in state $\underline{s}$ such that $\bar{c} \geq \underline{c}$. It follows that $x(i, C) \leq 1$ while $x(j, M) \leq 1 \leq x(j, Z-C)$. Then, since individual rationality requires $x(i, C) \leq x(j, Z-C) \leq x(j, M)$ whenever $x(i, C) \leq$ $x(j, M)$, we have a contradiction. Therefore, we know that $Z-C, Z-\widetilde{C} \in \mathbf{C}^{+}$, for all candidate contracts. Now part (i) obtains directly from the assumption $C, D \in \mathbf{C}^{-}$, which guarantees that $\bar{d} \leq \bar{c} \leq \underline{c} \leq \underline{d}$ if and only if $\bar{z}-\bar{d} \geq \bar{z}-\bar{c} \geq \underline{z}-\underline{c} \geq \underline{z}-\underline{d}$. Similarly, part (ii) is now a direct consequence of the assumption $C, D \in \mathbf{C}^{+}$, which guarantees that $\bar{d} \geq \bar{c} \geq \underline{c} \geq \underline{d}$ if and only if $\bar{z}-\bar{c} \geq \bar{z}-\bar{d} \geq \underline{z}-\underline{d} \geq \underline{z}-\underline{c}$.

Proof of Proposition 2. The assumption $\varepsilon \geq 0$ means that both endowments $M$ and $W$ belong to $\mathbf{C}^{+}$, which in turn implies that the same is true for all candidate contracts. Then, from Lemma 2 we know that a positive transfer results in a reduction in risk on one side of the market and an increase in risk on the other side. Formally, if $x(i, W)>x(j, M)$ then $W \succsim_{S C} C_{i j}(v ; \beta, \varepsilon)$ and $Z-C_{i j}(v ; \beta, \varepsilon) \succsim_{S C} M$, and if $x(i, M) \leq x(j, M)$ then $W \precsim_{S C}$ $C_{i j}(v ; \beta, \varepsilon)$ and $Z-C_{i j}(v ; \beta, \varepsilon) \precsim_{S C} M$.

Assume for now that $x(i, W)-x(j, M) \geq 0$ for all $\langle i, j\rangle$. This implies that the relevant $\mathrm{PC}$ for women is (4) in all couples $\langle i, j\rangle$. Then, by Lemma 1, Ms $i^{\prime}$ has an advantage over Ms $i^{\prime \prime}$, while Mr $j^{\prime \prime}$ has an advantage over Mr $j^{\prime}$. To show GDD in this case, we proceed analogously to Lemma 3.

If $C_{i^{\prime} j^{\prime}}\left(v^{\prime} ; \beta, \varepsilon\right)$ satisfies the PKC of Mr $j^{\prime \prime}$, we have GDD directly, since Ms $i^{\prime}$ cannot do worse than $C_{i^{\prime} j^{\prime}}\left(v^{\prime} ; \beta, \varepsilon\right)$ when she faces the less stringent $\mathrm{PC}(5)$ of $\mathrm{Mr} j^{\prime \prime}$.

Suppose instead that $C_{i^{\prime} j^{\prime}}\left(v^{\prime} ; \beta, \varepsilon\right)$ violates the $\mathrm{PKC}$ of $\mathrm{Mr} j^{\prime \prime}$. We first show that $C_{i^{\prime \prime} j^{\prime \prime}}\left(v^{\prime \prime} ; \beta, \varepsilon\right)$ is more Jewitt-risky than $C_{i^{\prime} j^{\prime}}\left(v^{\prime} ; \beta, \varepsilon\right)$ in this case. Assume indirectly the opposite. Then, by the revealed preference of Ms $i^{\prime \prime}$ for $\operatorname{Mr} j^{\prime \prime}$, the contract $C_{i^{\prime \prime} j^{\prime \prime}}\left(v^{\prime \prime} ; \beta, \varepsilon\right)$ 
satisfies the PKC of Mr $j^{\prime \prime}$ while either the PC (5) or the $\mathrm{PKC}$ of $\mathrm{Mr} j^{\prime}$ must be binding or violated. The PKCs of $\mathrm{Mr} j^{\prime}$ and $j^{\prime \prime}$ cross at most once on $\mathbf{C}^{+}$. Moreover, the PC (5) of $\operatorname{Mr} j^{\prime}$ and the $\mathrm{PKC}$ of $\mathrm{Mr} j^{\prime \prime}$ also cross at most once on $\mathbf{C}^{+}$, since the former can be expressed as a pivoted, steeper PKC of $\mathrm{Mr} j^{\prime}$, which reinforces the single-crossing property of two PKCs. Therefore, any contract which is more Jewitt-risky than $C_{i^{\prime \prime} j^{\prime \prime}}\left(v^{\prime \prime} ; \beta, \varepsilon\right)$ and satisfies both the $\mathrm{PC}(5)$ and the PKC of $\mathrm{Mr} j^{\prime}$ must also satisfy the PKC of Mr $j^{\prime \prime}$. However, since this set of contracts includes $C_{i^{\prime} j^{\prime}}\left(v^{\prime} ; \beta, \varepsilon\right)$ by our indirect assumption, we have a contradiction to our initial assumption that $C_{i^{\prime} j^{\prime}}\left(v^{\prime} ; \beta, \varepsilon\right)$ violates the $\mathrm{PKC}$ of $\mathrm{Mr} j^{\prime \prime}$.

Now, assume that Ms $i^{\prime}$ could improve upon $C_{i^{\prime \prime} j^{\prime \prime}}\left(v^{\prime \prime} ; \beta, \varepsilon\right)$ by the less Jewitt-risky contract $C_{i^{\prime} j^{\prime}}\left(v^{\prime} ; \beta, \varepsilon\right)$. Since $C_{i^{\prime \prime} j^{\prime \prime}}\left(v^{\prime \prime} ; \beta, \varepsilon\right)$ satisfies the $\mathrm{PC}(5)$ of $\mathrm{Mr} j^{\prime \prime}$, any reduced spread which is preferred by Ms $i^{\prime}$ must also be preferred by Ms $i^{\prime \prime}$. Moreover, the PC of Ms $i^{\prime \prime}$ cannot be violated by a reduced spread which does not reduce her expected utility, hence $C_{i^{\prime} j^{\prime}}\left(v^{\prime} ; \beta, \varepsilon\right)$ must also satisfy the PC (4) of Ms $i^{\prime \prime}$. But since Ms $i^{\prime \prime}$ revealed that such a contract cannot be better than $C_{i^{\prime \prime} j^{\prime \prime}}\left(v^{\prime \prime} ; \beta, \varepsilon\right)$, we obtain $C_{i^{\prime \prime} j^{\prime \prime}}\left(v^{\prime \prime} ; \beta, \varepsilon\right) \succsim_{i^{\prime \prime}} C_{i^{\prime} j^{\prime}}\left(v^{\prime} ; \beta, \varepsilon\right)$. Then, by Jewitt's order, we have $C_{i^{\prime \prime} j^{\prime \prime}}\left(v^{\prime \prime} ; \beta, \varepsilon\right) \succsim_{i^{\prime}} C_{i^{\prime} j^{\prime}}\left(v^{\prime} ; \beta, \varepsilon\right)$. Further, since Ms $i^{\prime}$ has an advantage over Ms $i^{\prime \prime}$, we obtain $C_{i^{\prime} j^{\prime \prime}}\left(v^{\prime \prime} ; \beta, \varepsilon\right) \succsim_{i^{\prime}} C_{i^{\prime} j^{\prime}}\left(v^{\prime} ; \beta, \varepsilon\right)$ and GDD.

Next we relax the assumption that $x(i, W)-x(j, M) \geq 0$ for all couples $\langle i, j\rangle$. Consider first the case where $x(i, W)-x(j, M) \leq 0$ for all $\langle i, j\rangle$. We can state the analogue of GDD when men propose a contract to women as a sufficient condition for NAM. Proceeding as above, we obtain that $\mathrm{Mr} j^{\prime}$ is willing and able to outbid $\mathrm{Mr} j^{\prime \prime}$ whenever the lifetime utility promises to women are such that $\mathrm{Mr} j^{\prime \prime}$ prefers Ms $i^{\prime \prime}$.

What remains to be shown is that GDD also holds in all cases where the sign of $x(i, W)-$ $x(j, M)$ varies across couples. From Lemma 7 in Appendix B we know that as $\varepsilon$ increases from 0 to 1 , the first couple to switch (from positive to negative sign) is $\left\langle i^{\prime \prime}, j^{\prime}\right\rangle$ and the last is $\left\langle i^{\prime}, j^{\prime \prime}\right\rangle$. This implies that if there is variation in the sign of $x(i, W)-x(j, M)$, these couples are among those who have opposite signs, i.e., it must be that $x\left(i^{\prime}, W\right)-x\left(j^{\prime \prime}, M\right) \geq 0$ and $x\left(i^{\prime \prime}, W\right)-x\left(j^{\prime}, M\right) \leq 0$.

Consider first the case where $x\left(i^{\prime}, W\right)-x\left(j^{\prime}, M\right) \geq 0$. In this case, Ms $i^{\prime}$ makes a nonnegative transfer in state $\underline{s}$ for all $j$ and her relevant PC is (4). Consider first the subcase where $x\left(i^{\prime \prime}, W\right)-x\left(j^{\prime \prime}, M\right) \leq 0$. That is, Ms $i^{\prime \prime}$ makes a non-negative transfer in state $\bar{s}$ and her relevant $\mathrm{PC}$ is $\mathrm{Ms}(3), \forall j$. Then, we know that $C_{i^{\prime \prime} j}(v ; \beta, \varepsilon) \succsim_{S C} W \succsim_{S C} C_{i^{\prime} j}(v ; \beta, \varepsilon)$, $\forall j$. Further, considering both men sharing risk with Ms $i^{\prime \prime}$, from Lemma 2 we know that $Z-C_{i^{\prime \prime} j}(v ; \beta, \varepsilon) \precsim_{S C} M, \forall j$, and in turn Lemma 1 implies that Mr $j^{\prime}$ has an advantage. Therefore, the preference of Ms $i^{\prime \prime}$ for Mr $j^{\prime \prime}$ reveals that the PKC of Mr $j^{\prime}$ cannot be slack at $C_{i^{\prime \prime} j^{\prime \prime}}\left(v^{\prime \prime} ; \beta, \varepsilon\right)$. Therefore, $C_{i^{\prime \prime} j^{\prime \prime}}\left(v^{\prime \prime} ; \beta, \varepsilon\right)$ is more Jewitt-risky than the single-crossing point 
of the two PKCs. As a result, for any $C$ such that $C_{i^{\prime \prime} j^{\prime \prime}}\left(v^{\prime \prime} ; \beta, \varepsilon\right) \succsim_{S C} C$, we know that $C$ must also be more Jewitt-risky than the single crossing point. Hence, if $C$ satisfies the PKC of $\mathrm{Mr} j^{\prime}$, then it satisfies the PKC of Mr $j^{\prime \prime}$ too. But since $C_{i^{\prime \prime} j^{\prime \prime}}\left(v^{\prime \prime} ; \beta, \varepsilon\right) \succsim_{S C} W_{S C}$ $C_{i^{\prime} j^{\prime}}\left(v^{\prime} ; \beta, \varepsilon\right)$, we know that the PKC of $\mathrm{Mr} j^{\prime \prime}$ must be slack at $C_{i^{\prime} j^{\prime}}\left(v^{\prime} ; \beta, \varepsilon\right)$. Hence we have $C_{i^{\prime} j^{\prime \prime}}\left(v^{\prime \prime} ; \beta, \varepsilon\right) \succsim_{i^{\prime}} C_{i^{\prime} j^{\prime}}\left(v^{\prime} ; \beta, \varepsilon\right)$, i.e., GDD.

Second, consider the subcase where $x\left(i^{\prime \prime}, W\right)-x\left(j^{\prime \prime}, M\right) \geq 0$. In this case, $C_{i^{\prime \prime} j^{\prime}}\left(v^{\prime} ; \beta, \varepsilon\right) \succsim_{S C}$ $W \succsim_{S C} C_{i^{\prime \prime} j^{\prime \prime}}\left(v^{\prime \prime} ; \beta, \varepsilon\right)$ while $C_{i^{\prime} j}\left(v^{\prime} ; \beta, \varepsilon\right) \precsim_{S C} W, \forall j$. First note that Ms $i^{\prime \prime}$ and $\operatorname{Mr} j^{\prime}$ can only mutually prefer a contract to autarky which is less Jewitt-risky than $W$, since $x\left(i^{\prime \prime}, W\right)-x\left(j^{\prime}, M\right) \leq 0$. At the same time, we have $W \succsim_{S C} C_{i^{\prime \prime} j^{\prime \prime}}\left(v^{\prime \prime} ; \beta, \varepsilon\right)$. Further, by individual rationality of $C_{i^{\prime \prime} j^{\prime \prime}}\left(v^{\prime \prime} ; \beta, \varepsilon\right)$, we have $C_{i^{\prime \prime} j^{\prime \prime}}\left(v^{\prime \prime} ; \beta, \varepsilon\right) \succsim_{i^{\prime \prime}} W$. Hence it must be that $M \succsim_{j^{\prime}} Z-C_{i^{\prime \prime} j^{\prime \prime}}\left(v^{\prime \prime} ; \beta, \varepsilon\right)$. As a result, the PKC of Mr $j^{\prime}$ cannot be slack at $C_{i^{\prime \prime} j^{\prime \prime}}\left(v^{\prime \prime} ; \beta, \varepsilon\right)$. Consider first any $C_{i^{\prime} j^{\prime}}\left(v^{\prime} ; \beta, \varepsilon\right)$ which is more Jewitt-risky than $C_{i^{\prime \prime} j^{\prime \prime}}\left(v^{\prime \prime} ; \beta, \varepsilon\right)$. In this case, the single-crossing property of PKCs implies that $C_{i^{\prime} j^{\prime}}\left(v^{\prime} ; \beta, \varepsilon\right)$ must also satisfy the PKC of Mr $j^{\prime \prime}$. Further, from Lemma 2 we know that $Z-C_{i^{\prime} j^{\prime}}\left(v^{\prime} ; \beta, \varepsilon\right) \succsim_{S C} M$, and in turn Lemma 1 implies that $\mathrm{Mr} j^{\prime \prime}$ has an advantage, and we have GDD. Alternatively, assume that $C_{i^{\prime} j^{\prime}}\left(v^{\prime} ; \beta, \varepsilon\right)$ is less Jewitt-risky than $C_{i^{\prime \prime} j^{\prime \prime}}\left(v^{\prime \prime} ; \beta, \varepsilon\right)$. However, we know that Ms $i^{\prime \prime}$ and $\mathrm{Mr} j^{\prime}$ cannot mutually prefer $C_{i^{\prime} j^{\prime}}\left(v^{\prime} ; \beta, \varepsilon\right)$ to autarky since $x\left(i^{\prime}, W\right)-x\left(j^{\prime}, M\right)$ and $x\left(i^{\prime \prime}, W\right)-x\left(j^{\prime}, M\right)$ do not have the same sign. Moreover, by individual rationality, $C_{i^{\prime} j^{\prime}}\left(v^{\prime} ; \beta, \varepsilon\right)$ cannot be worse than autarky for $\operatorname{Mr} j^{\prime}$. Therefore $W \succsim_{i^{\prime \prime}} C_{i^{\prime} j^{\prime}}\left(v^{\prime} ; \beta, \varepsilon\right)$. However, by individual rationality of the couple $\left\langle i^{\prime \prime}, j^{\prime \prime}\right\rangle$, we know that $C_{i^{\prime \prime} j^{\prime \prime}}\left(v^{\prime \prime} ; \beta, \varepsilon\right) \succsim_{i^{\prime \prime}}$ $W$. Hence, $C_{i^{\prime \prime} j^{\prime \prime}}\left(v^{\prime \prime} ; \beta, \varepsilon\right) \succsim_{i^{\prime \prime}} W \succsim_{i^{\prime \prime}} C_{i^{\prime} j^{\prime}}\left(v^{\prime} ; \beta, \varepsilon\right)$. Using Jewitt's order, this implies $C_{i^{\prime \prime} j^{\prime \prime}}\left(v^{\prime \prime} ; \beta, \varepsilon\right) \succsim_{i^{\prime}} C_{i^{\prime} j^{\prime}}\left(v^{\prime} ; \beta, \varepsilon\right)$, and we have GDD.

Consider now the case where $x\left(i^{\prime}, W\right)-x\left(j^{\prime}, M\right) \leq 0$, that is, Ms $i^{\prime}$ switches the state of transfer between $\operatorname{Mr} j^{\prime}$ and $\operatorname{Mr} j^{\prime \prime}$. Consider first the subcase where $x\left(i^{\prime \prime}, W\right)-x\left(j^{\prime \prime}, M\right) \leq 0$, i.e., Ms $i^{\prime \prime}$ makes a non-negative transfer in state $\bar{s}, \forall j$. This proof is analogous to the previous case. The remaining subcase concerns $x\left(i^{\prime \prime}, W\right)-x\left(j^{\prime \prime}, M\right) \geq 0$. In this case, both women make a non-negative transfer in state $\underline{s}$ when sharing risk with $\mathrm{Mr} j^{\prime \prime}$, and in state $\bar{s}$ when with Mr $j^{\prime}$. That is, women's consumption is more (less) Jewitt-risky than their income when with $\operatorname{Mr} j^{\prime \prime}\left(\operatorname{Mr} j^{\prime}\right)$. Notice that GDD is logically equivalent to:

$$
C_{i^{\prime} j^{\prime}}\left(v^{\prime} ; \beta, \varepsilon\right) \succ_{i^{\prime}} C_{i^{\prime} j^{\prime \prime}}\left(v^{\prime \prime} ; \beta, \varepsilon\right) \quad \Rightarrow \quad C_{i^{\prime \prime} j^{\prime}}\left(v^{\prime} ; \beta, \varepsilon\right) \succ_{i^{\prime \prime}} C_{i^{\prime \prime} j^{\prime \prime}}\left(v^{\prime \prime} ; \beta, \varepsilon\right) .
$$

Define $C^{\prime \prime}$ as follows: if $C_{i^{\prime} j^{\prime \prime}}\left(v^{\prime \prime} ; \beta, \varepsilon\right) \succsim_{i^{\prime}} C_{i^{\prime \prime} j^{\prime \prime}}\left(v^{\prime \prime} ; \beta, \varepsilon\right)$ then $C_{i^{\prime} j^{\prime \prime}}\left(v^{\prime \prime} ; \beta, \varepsilon\right) \sim_{i^{\prime}} C^{\prime \prime} \sim_{i^{\prime \prime}}$ $C_{i^{\prime \prime} j^{\prime}}\left(v^{\prime} ; \beta, \varepsilon\right)$, otherwise $C^{\prime \prime}=C_{i^{\prime} j^{\prime \prime}}\left(v^{\prime \prime} ; \beta, \varepsilon\right)$. Analogously, define $C^{\prime}$ as follows: if $C_{i^{\prime} j^{\prime}}\left(v^{\prime} ; \beta, \varepsilon\right) \succsim_{i^{\prime}}$ $C_{i^{\prime \prime} j^{\prime}}\left(v^{\prime} ; \beta, \varepsilon\right)$ then $C_{i^{\prime} j^{\prime}}\left(v^{\prime} ; \beta, \varepsilon\right) \sim_{i^{\prime}} C^{\prime} \sim_{i^{\prime \prime}} C_{i^{\prime \prime} j^{\prime}}\left(v^{\prime} ; \beta, \varepsilon\right)$, otherwise $C^{\prime}=C_{i^{\prime} j^{\prime}}\left(v^{\prime} ; \beta, \varepsilon\right)$. Then we also know that $C^{\prime} \succsim_{S C} W \succsim_{S C} C^{\prime \prime}$. Ms $i^{\prime}$ revealed $C^{\prime} \succ_{i^{\prime}} C^{\prime \prime}$ by the premise 
$C_{i^{\prime} j^{\prime}}\left(v^{\prime} ; \beta, \varepsilon\right) \succ_{i^{\prime}} C_{i^{\prime} j^{\prime \prime}}\left(v^{\prime \prime} ; \beta, \varepsilon\right)$. Hence, by Jewitt's order, Ms $i^{\prime \prime}$ agrees, i.e., $C^{\prime} \succ_{i^{\prime \prime}} C^{\prime \prime}$. Moreover, since Ms $i^{\prime}$ has an advantage when with $\mathrm{Mr} j^{\prime \prime}$, while Ms $i^{\prime \prime}$ has an advantage when with Mr $j^{\prime}$, we obtain $C_{i^{\prime \prime} j^{\prime}}\left(v^{\prime} ; \beta, \varepsilon\right) \succsim_{i^{\prime \prime}} C^{\prime} \succ_{i^{\prime \prime}} C^{\prime \prime} \succsim_{i^{\prime \prime}} C_{i^{\prime \prime} j^{\prime \prime}}\left(v^{\prime \prime} ; \beta, \varepsilon\right)$, and hence GDD holds. Therefore, the economy satisfies NAM.

Proof of Lemma 3. We show that GID, a sufficient condition for PAM, holds. First, note that $C \in \mathbf{C}^{-}$implies that $W \in \mathbf{C}^{-}$, i.e., that $\varepsilon \leq 0$. Then, by Lemma 6 in Appendix B we know that women's relevant PC is (4). Second, from Lemma 2, we know that $C \succsim_{S C} W$ and $Z-C \succsim_{S C} M$. In this case, by Lemma 1, we know that Ms $i^{\prime \prime}$ (Mr $j^{\prime \prime}$ ) has an advantage over Ms $i^{\prime}\left(\operatorname{Mr} j^{\prime}\right)$.

Further, the PKCs (2) of Mr $j^{\prime}$ and $\mathrm{Mr} j^{\prime \prime}$ satisfy the following single-crossing property. Let $C$ satisfy (2) for both $\operatorname{Mr} j^{\prime}$ and $\operatorname{Mr} j^{\prime \prime}$. Any contract $\widetilde{C}$ which is less Jewitt-risky for the man, i.e., $Z-\widetilde{C} \succsim_{S C} Z-C$, and which satisfies the PKC for Mr $j^{\prime}$ must also satisfy the PKC for Mr $j^{\prime \prime}$. Conversely, any contract $C^{\prime \prime}$ which is more Jewitt-risky for the man, i.e. $Z-C^{\prime \prime} \precsim_{S C} Z-C$, and which satisfies the PKC for $\mathrm{Mr} j^{\prime \prime}$ must also satisfy the PKC for $\operatorname{Mr} j^{\prime}$.

Suppose first that $C_{i^{\prime \prime} j^{\prime}}\left(v^{\prime} ; \beta, \varepsilon\right)$ satisfies the $\mathrm{PKC}$ of $\mathrm{Mr} j^{\prime \prime}$. Then we have GID directly, since Ms $i^{\prime \prime}$ cannot do worse than $C_{i^{\prime \prime} j^{\prime}}\left(v^{\prime} ; \beta, \varepsilon\right)$ when she faces the less stringent PC (5) of $\operatorname{Mr} j^{\prime \prime}$.

Now, suppose instead that $C_{i^{\prime \prime} j^{\prime}}\left(v^{\prime} ; \beta, \varepsilon\right)$ violates the $\mathrm{PKC}$ of $\mathrm{Mr} j^{\prime \prime}$. We first show that $C_{i^{\prime} j^{\prime \prime}}\left(v^{\prime \prime} ; \beta, \varepsilon\right)$ is less Jewitt-risky than $C_{i^{\prime \prime} j^{\prime}}\left(v^{\prime} ; \beta, \varepsilon\right)$. Assume indirectly the opposite. By the revealed preference of $\mathrm{Ms} i^{\prime}$ for $\mathrm{Mr} j^{\prime \prime}$, the contract $C_{i^{\prime} j^{\prime \prime}}\left(v^{\prime \prime} ; \beta, \varepsilon\right)$ satisfies the $\mathrm{PKC}$ of $\mathrm{Mr} j^{\prime \prime}$ while either the PC (5) of $\mathrm{Mr} j^{\prime}$ or the PKC of $\mathrm{Mr} j^{\prime}$ must be binding or violated. The PKCs of $\mathrm{Mr} j^{\prime}$ and $\mathrm{Mr} j^{\prime \prime}$ cross at most once on $\mathbf{C}^{-}$. Moreover, the $\mathrm{PC}$ (5) of $\mathrm{Mr} j^{\prime}$ and the PKC of $\mathrm{Mr} j^{\prime \prime}$ also cross at most once on $\mathbf{C}^{-}$, since the former can be expressed as a pivoted PKC of $\mathrm{Mr} j^{\prime}$ with an even steeper slope, which reinforces the single-crossing property present among two PKCs. Therefore, any contract which is less Jewitt-risky than $C_{i^{\prime} j^{\prime \prime}}\left(v^{\prime \prime} ; \beta, \varepsilon\right)$ and which satisfies both the PC (5) and the PKC of Mr $j^{\prime}$ also satisfies the PKC of Mr $j^{\prime \prime}$. In particular, this is the case for $C_{i^{\prime \prime} j^{\prime}}\left(v^{\prime} ; \beta, \varepsilon\right)$. However, this contradicts our assumption that $C_{i^{\prime \prime} j^{\prime}}\left(v^{\prime} ; \beta, \varepsilon\right)$ violates the $\mathrm{PKC}$ of $\mathrm{Mr} j^{\prime \prime}$.

Now, assume that Ms $i^{\prime \prime}$ could improve upon $C_{i^{\prime} j^{\prime \prime}}\left(v^{\prime \prime} ; \beta, \varepsilon\right)$ by the more Jewitt-risky contract $C_{i^{\prime \prime} j^{\prime}}\left(v^{\prime} ; \beta, \varepsilon\right)$. Since $C_{i^{\prime} j^{\prime \prime}}\left(v^{\prime \prime} ; \beta, \varepsilon\right)$ satisfies the PC of Ms $i^{\prime}$, any increased spread which is preferred by Ms $i^{\prime \prime}$ must also be preferred by Ms $i^{\prime}$. Moreover, the PC of Ms $i^{\prime}$ cannot be violated by an increased spread which does not reduce expected utility, hence $C_{i^{\prime \prime} j^{\prime}}\left(v^{\prime} ; \beta, \varepsilon\right)$ must also satisfy the PC (4) of Ms $i^{\prime}$. But since Ms $i^{\prime}$ revealed that such a contract cannot 
be better than $C_{i^{\prime \prime} j^{\prime}}\left(v^{\prime} ; \beta, \varepsilon\right)$, we obtain $C_{i^{\prime} j^{\prime \prime}}\left(v^{\prime \prime} ; \beta, \varepsilon\right) \succsim_{i^{\prime}} C_{i^{\prime \prime} j^{\prime}}\left(v^{\prime} ; \beta, \varepsilon\right)$. Then, by Jewitt's order, we obtain $C_{i^{\prime} j^{\prime \prime}}\left(v^{\prime \prime} ; \beta, \varepsilon\right) \succsim_{i^{\prime \prime}} C_{i^{\prime \prime} j^{\prime}}\left(v^{\prime} ; \beta, \varepsilon\right)$. Finally, since Ms $i^{\prime \prime}$ has an advantage over Ms $i^{\prime}$, we obtain $C_{i^{\prime \prime} j^{\prime \prime}}\left(v^{\prime \prime} ; \beta, \varepsilon\right) \succsim_{i^{\prime \prime}} C_{i^{\prime \prime} j^{\prime}}\left(v^{\prime} ; \beta, \varepsilon\right)$ and GID.

Proof of Proposition 3. If $\varepsilon=-1$, transfers cannot be larger than those which provide risk-free consumption to the woman. Therefore, we know that $C_{i j}(v ; \beta, \varepsilon) \in \mathbf{C}^{-}$for all $v$ and for all $\langle i, j\rangle$. Then, using Lemma 3 we obtain PAM.

Proof of Proposition 4. In order to determine whether the PCs of spouses in a couple are stringent enough to guarantee $C_{i j}(v ; \beta, \varepsilon) \in \mathbf{C}^{-}$for all $v$ and for all $\langle i, j\rangle$, we consider both the minimum sure consumption of a woman which satisfies her PC, and the maximum sure consumption of a woman which satisfies her partner's PC.

Definition 6. Consider $\varepsilon<0$. Call $c_{i}(\beta, \varepsilon)\left(c_{j}(\beta, \varepsilon)\right)$ the level of constant consumption by Ms $i$ which satisfies her $P C$, (4) (the $P C$ of $M r j$, (5)), as equality. In mathematical terms, $c_{i}(\beta, \varepsilon)$ is implicitly given by

$$
\frac{1}{1-\beta} u\left(i, c_{i}(\beta, \varepsilon)\right)=u(i, 1-\varepsilon y)+\frac{\beta}{1-\beta} \mathbb{E} u(i, W),
$$

and $c_{j}(\beta, \varepsilon)$ is defined by

$$
u\left(j, \bar{z}-c_{j}(\beta, \varepsilon)\right)+\frac{\beta}{1-\beta} \mathbb{E} u\left(j, Z-c_{j}(\beta, \varepsilon)\right)=u(j, 1+y)+\frac{\beta}{1-\beta} \mathbb{E} u(j, M) .
$$

Note that $c_{i}(\beta, \varepsilon)$ can be either above or below $c_{j}(\beta, \varepsilon)$. If $c_{i}(\beta, \varepsilon)>c_{j}(\beta, \varepsilon)$, then the couple $\langle i, j\rangle$ cannot sustain transfers that are large enough to provide risk-free consumption to Ms $i$.

We can now restate our sufficient condition for PAM in Lemma 3 using $c_{i}(\beta, \varepsilon)$ and $c_{j}(\beta, \varepsilon)$.

Lemma 4. For any $-1<\varepsilon<0, c_{i}(\beta, \varepsilon) \geq c_{j}(\beta, \varepsilon)$ if and only if $C_{i j}(v ; \beta, \varepsilon) \in \mathbf{C}^{-}$for all $v$.

Proof. First, note that any contract which assigns less consumption than $c_{i}(\beta, \varepsilon)$ in both states violates the woman's PC (4) and any contract which assigns more than $c_{j}(\beta, \varepsilon)$ in both states violates the man's PC (5). Second, note that $\varepsilon<0$ means $W \in \mathbf{C}^{-}$. Now, consider first the case where $c_{j}(\beta, \varepsilon)=c_{i}(\beta, \varepsilon)$. Then we know that the crossing point of the two PCs is exactly on the line $\mathbf{C}^{-} \cap \mathbf{C}^{+}$, and any contract in $\mathbf{C}^{+} \backslash \mathbf{C}^{-}$violates at least one PC. Second, suppose that $c_{j}(\beta, \varepsilon)<c_{i}(\beta, \varepsilon)$. Then the woman cannot even get to constant consumption, because the $\mathrm{PC}$ of the man crosses the line $\mathbf{C}^{-} \cap \mathbf{C}^{+}$below her own $\mathrm{PC}$, which implies $C_{i j}(v ; \beta, \varepsilon) \in \mathbf{C}^{-}$. 
To show the converse, first note that for any $-1<\varepsilon<0, C_{i j}(v ; \beta, \varepsilon) \in \mathbf{C}^{-}$for all $v$ implies for that the couple $\langle i, j\rangle$ cannot sustain efficient transfers for any $v$. Consider first the case where the couple cannot sustain positive transfers. The man's PC is always violated at any $C \in \mathbf{C}^{-} \cap \mathbf{C}^{+}$for which the $\mathrm{PC}$ of the woman is satisfied. Therefore, $c_{j}(\beta, \varepsilon) \leq c_{i}(\beta, \varepsilon)$. Second, assume that the couple can sustain positive transfers. Then there exists a promise $v^{L R}$ such that both the man's $\mathrm{PC}$ and the woman's PC hold as equality. Further, the contract $C_{i j}\left(v^{L R} ; \beta, \varepsilon\right)$ corresponds to the intersection of the two PCs. However, since we have $C_{i j}(v ; \beta, \varepsilon) \in \mathbf{C}^{-}$for all $v$ by assumption, we know that $C_{i j}\left(v^{L R} ; \beta, \varepsilon\right) \in \mathbf{C}^{-}$. Therefore, since the two PCs intersect in $\mathbf{C}^{-}$, we obtain $c_{j}(\beta, \varepsilon) \leq c_{i}(\beta, \varepsilon)$.

Next we prove comparative statics results for $c_{i}(\beta, \varepsilon)$ and $c_{j}(\beta, \varepsilon)$ as $\beta$, risk aversion, and $\varepsilon$ change. $^{20}$

Lemma 5. Assume $\varepsilon<0$.

- $c_{i}(\beta, \varepsilon)$ is decreasing in $\beta$ for all $i$,

- $c_{i^{\prime}}(\beta, \varepsilon) \geq c_{i^{\prime \prime}}(\beta, \varepsilon)$ for all $\beta$, and

- $c_{i}(\beta, \varepsilon)$ is increasing (decreasing) in $\varepsilon$ if $\varepsilon \leq \varepsilon_{i}(\beta)$ (if $\varepsilon \geq \varepsilon_{i}(\beta)$ ), where $\varepsilon_{i}(\beta)$ is implicitly defined by $x\left(i, 1+\varepsilon_{i}(\beta) y\right)=(1-\beta \operatorname{Pr}(\bar{s})) / \beta \operatorname{Pr}(\bar{s})$.

Similarly,

- $c_{j}(\beta, \varepsilon)$ is increasing in $\beta$ for all $j$,

- $c_{j^{\prime \prime}}(\beta, \varepsilon) \geq c_{j^{\prime}}(\beta, \varepsilon)$ for all $\beta$, and

- $c_{j}(\beta, \varepsilon)$ is increasing (decreasing) in $\varepsilon$ if $\varepsilon \leq \varepsilon_{j}(\beta)$ (if $\varepsilon \geq \varepsilon_{j}(\beta)$ ), where $\varepsilon_{j}(\beta)$ is implicitly defined by $x\left(j, Z-c_{j}\left(\beta, \varepsilon_{j}(\beta)\right)\right)=\beta \operatorname{Pr}(\underline{s}) /(1-\beta \operatorname{Pr}(\underline{s}))$.

Proof. Multiplying the PC (4) by $(1-\beta)$, the level $c_{i}(\beta, \varepsilon)$ is defined by $u\left(i, c_{i^{\prime \prime}}(\beta, \varepsilon)\right)=$ $\alpha u(i, \bar{w})+(1-\alpha) u(i, \underline{w})$ where $\alpha=\beta \operatorname{Pr}(\bar{s})$. Under the assumption $\varepsilon<0$, we have $\underline{w} \geq \bar{w}$, and the right-hand side of the equality decreases with $\beta$, hence the monotonicity of $c_{i}$ in the first argument.

Moreover, applying Jewitt's order, we get $u\left(i^{\prime \prime}, c_{i^{\prime}}(\beta, \varepsilon)\right) \geq \alpha u\left(i^{\prime \prime}, \bar{w}\right)+(1-\alpha) u\left(i^{\prime \prime}, \underline{w}\right)$, since $\left(c_{i^{\prime}}(\beta, \varepsilon), c_{i^{\prime}}(\beta, \varepsilon)\right) \succsim_{S C} W$. This inequality implies that the PC (4) of Ms $i^{\prime \prime}$ is satisfied at $\left(c_{i^{\prime}}(\beta, \varepsilon), c_{i^{\prime}}(\beta, \varepsilon)\right.$, and we have $c_{i^{\prime \prime}}(\beta, \varepsilon) \leq c_{i^{\prime}}(\beta, \varepsilon)$.

To prove the hump-shaped relationship between $c_{i}(\beta, \varepsilon)$ and $\varepsilon$, note first that the righthand side of the woman's relevant PC, (4), $u(i, 1-\varepsilon y)+\frac{\beta}{1-\beta} \mathbb{E} u(i, W)$, is strictly concave

\footnotetext{
${ }^{20}$ Note that while $\varepsilon$ does not affect the man's continuation value in autarky, it determines the size of aggregate risk $(1+\varepsilon) Y$, which has to be borne in its entirety by the man, given that the woman's consumption is constant.
} 
in $\varepsilon$ due to the strict concavity of $u$ in consumption. Maximising it with respect to $\varepsilon$, the first-order condition is

$$
-\left(1+\frac{\beta}{1-\beta} \operatorname{Pr}(\underline{s})\right) \frac{\partial}{\partial c} u(i, 1-\varepsilon y) y+\left(\operatorname{Pr}(\bar{s}) \frac{\beta}{1-\beta}\right) \frac{\partial}{\partial c} u(i, 1+\varepsilon y) y=0 .
$$

Due to the Inada conditions and concavity, the first-order condition is necessary and sufficient for a maximum. Rearranging, the maximiser is characterised by $\frac{u_{c}\left(i, 1+\varepsilon_{i}(\beta) y\right)}{u_{c}\left(i, 1-\varepsilon_{i}(\beta) y\right)}=\frac{1-\beta \operatorname{Pr}(\bar{s})}{\beta \operatorname{Pr}(\bar{s})}$. Since $c_{i}(\beta, \varepsilon)$ increases with the continuation value of autarky, the result follows.

Now, proceeding analogously for men, we obtain $c_{j}(\beta, \varepsilon)$ by multiplying $(5)$ by $(1-\beta)$, where $\left(1-\alpha^{\prime}\right) u\left(j, \bar{z}-c_{j}(\beta, \varepsilon)\right)+\alpha^{\prime} u\left(j, \underline{z}-c_{j}(\beta, \varepsilon)\right)=\left(1-\alpha^{\prime}\right) u(j, \bar{m})+\alpha^{\prime} u(j, \underline{m})$ and $\alpha^{\prime}=$ $\beta \operatorname{Pr}(\underline{s})$. The monotonicity with respect to $\beta$ obtains since both $\bar{z} \geq \underline{z}$ and $\bar{w} \geq \underline{w}$ while $\alpha^{\prime}$ increases in $\beta$.

Moreover, since $W \in \mathbf{C}^{-}$and $\left(c_{i^{\prime}}(\beta, \varepsilon), c_{i^{\prime}}(\beta, \varepsilon)\right)$ is a reduced spread of $W$, Lemma 2 says that $Z-c_{j^{\prime}}(\beta, \varepsilon) \succeq_{S C} M$. Applying Jewitt's order to the PC (5) of Mr $j^{\prime}$ and $\mathrm{Mr} j^{\prime \prime}$, we obtain $c_{j^{\prime \prime}}(\beta, \varepsilon) \geq c_{j^{\prime}}(\beta, \varepsilon)$ for all $\beta$.

Finally, we prove the hump-shaped relationship between $c_{j}(\beta, \varepsilon)$ and $\varepsilon$. First, we show that $\varepsilon_{j}(\beta)$ maximises $c_{j}(\beta, \varepsilon)$. To do so, we need to show that $c_{j}\left(\beta, \varepsilon_{j}(\beta)\right)$ violates the $\mathrm{PC}$ (5) for all $\varepsilon \neq \varepsilon_{j}(\beta)$. If the woman has a risk-free level of consumption $k$, then the left-hand side of (5) can be expressed by the function

$\tilde{v}(k, \varepsilon)=\left[1+\frac{\beta}{1-\beta} \operatorname{Pr}(\bar{s})\right] u(j, 2+(1+\varepsilon) y-k)+\frac{\beta}{1-\beta}[1-\operatorname{Pr}(\bar{s})] u(j, 2-(1+\varepsilon) y-k)$.

By definition (5) holds as equality at $k=c_{j}(\beta, \varepsilon)$ for all $\varepsilon$, hence $\tilde{v}\left(c_{j}(\beta, \varepsilon), \varepsilon\right)=\tilde{v}\left(c_{j}\left(\beta, \varepsilon^{\prime}\right), \varepsilon^{\prime}\right)$ for any $\varepsilon$ and $\varepsilon^{\prime}$. Moreover, if $\tilde{v}\left(c_{j}(\beta, \varepsilon), \varepsilon^{\prime}\right) \geq \tilde{v}\left(c_{j}(\beta, \varepsilon), \varepsilon\right)$, then $c_{j}\left(\beta, \varepsilon^{\prime}\right) \geq c_{j}(\beta, \varepsilon)$. Since $u$ is strictly concave in consumption, $\tilde{v}()$ is strictly concave in its second argument, $\varepsilon$. Setting the partial derivative of $\tilde{v}()$ with respect to the second argument equal to 0 evaluated at $k=c_{j}\left(\beta, \varepsilon_{j}(\beta)\right)$ yields the condition $\frac{u_{c}\left[j, 2+\left(1+\varepsilon_{j}(\beta)\right) y-c_{j}\left(\beta, \varepsilon_{j}(\beta)\right)\right]}{u_{c}\left[j, 2-\left(1+\varepsilon_{j}(\beta)\right) y-c_{j}\left(\beta, \varepsilon_{j}(\beta)\right)\right]}=\frac{\beta \operatorname{Pr}(\underline{s})}{1-\beta \operatorname{Pr}(\underline{s})}$, which is equivalent to $x\left(j, Z-c_{j}\left(\beta, \varepsilon_{j}(\beta)\right)\right)=\beta \operatorname{Pr}(\underline{s}) /(1-\beta \operatorname{Pr}(\underline{s}))$. Therefore, $\varepsilon_{j}(\beta)$ satisfies $\tilde{v}\left(c_{j}\left(\beta, \varepsilon_{j}(\beta)\right), \varepsilon\right)<\tilde{v}\left(c_{j}\left(\beta, \varepsilon_{j}(\beta)\right), \varepsilon_{j}(\beta)\right)=\tilde{v}\left(c_{j}(\beta, \varepsilon), \varepsilon\right)$ for all $\varepsilon \neq \varepsilon_{j}(\beta)$. As a result, $c_{j}\left(\beta, \varepsilon_{j}(\beta)\right)$ violates the $\mathrm{PC}(5)$ for all $\varepsilon \neq \varepsilon_{j}(\beta)$, hence $c_{j}\left(\beta, \varepsilon_{j}(\beta)\right)>c_{j}(\beta, \varepsilon)$ for all $\varepsilon \neq \varepsilon_{j}(\beta)$, as we wanted to show.

Now, given that $\tilde{v}()$ is strictly concave in $\varepsilon$, we know that $\tilde{v}(k, \varepsilon)$ in increasing (decreasing) with $\varepsilon$ whenever $\varepsilon \leq \varepsilon_{j}(\beta)\left(\varepsilon \geq \varepsilon_{j}(\beta)\right)$. This implies that $c_{j}(\beta, \varepsilon)$ has to increase (decrease) with $\varepsilon$ for $\varepsilon \leq \varepsilon_{j}(\beta)\left(\varepsilon \geq \varepsilon_{j}(\beta)\right)$ to keep $\tilde{v}\left(c_{j}(\beta, \varepsilon), \varepsilon\right)$ constant, which proves the result.

Intuitively, given $\varepsilon<0$, as agents become more patient, they find it less difficult to commit to large transfers. In turn, $c_{i}(\beta, \varepsilon)$ decreases as the woman's $\mathrm{PC}$ is less stringent, and $c_{j}(\beta, \varepsilon)$ 
increases as the man's PC is less stringent. Moreover, given that risk-free consumption by the woman cannot be more Jewitt-risky than her income, $c_{i^{\prime}}(\beta, \varepsilon)$ satisfies the PC (4) of Ms $i^{\prime \prime}$. As men's consumption is always less Jewitt-risky than their income if $\varepsilon<0, c_{j^{\prime}}(\beta, \varepsilon)$ satisfies the $\mathrm{PC}(5)$ of $\mathrm{Mr} j^{\prime \prime}$ as well. That is, these consumption levels capture advantage. Further, the woman's PC becomes more stringent if her outside option improves. That is, if an increase in $\varepsilon$ reduces (increases) the continuation value of autarky, then $c_{i}(\beta, \varepsilon)$ increases (decreases). We show that autarky utility is first increasing, then decreasing in $\varepsilon$. Finally, $c_{j}(\beta, \varepsilon)$ is also first increasing, then decreasing in $\varepsilon$. To see why, note that the man bears all aggregate risk when the woman's consumption is constant. An increase in $\varepsilon$ from -1 may therefore first increase $c_{j}(\beta, \varepsilon)$ if the low aggregate income state, $\underline{s}$, occurs with low enough probability. However, as $\varepsilon$ increases beyond a critical level, the negative effect of bearing a greater risk dominates and $c_{j}(\beta, \varepsilon)$ declines.

Now we can complete our proof. Lemma 5 says that $c_{i}(\beta, \varepsilon)$ decreases while $c_{j}(\beta, \varepsilon)$ increases in $\beta$ for any couple $\langle i, j\rangle$, including importantly $\langle I, J\rangle$. In addition, the two functions $c_{I}(\beta, \varepsilon)$ and $c_{J}(\beta, \varepsilon)$ are continuous in $\beta$ by our continuity assumptions on $u$. Since $c_{I}(0, \varepsilon)<$ $c_{J}(0, \varepsilon)$ and $c_{I}(1, \varepsilon)>c_{J}(1, \varepsilon)$ for any $\varepsilon<0$, there exists a $\bar{\beta}(\varepsilon) \in(0,1)$ such that $c_{I}(\bar{\beta}(\varepsilon), \varepsilon)=$ $c_{J}(\bar{\beta}(\varepsilon), \varepsilon)$ and $c_{I}(\beta, \varepsilon) \geq c_{J}(\beta, \varepsilon)$ if $\beta<\bar{\beta}(\varepsilon)$. Moreover, Lemma 5 implies that $c_{i}(\beta, \varepsilon) \geq$ $c_{j}(\beta, \varepsilon)$, for any $i \in \mathcal{I}$ and any $j \in \mathcal{J}$, whenever $c_{I}(\beta, \varepsilon) \geq c_{J}(\beta, \varepsilon)$. Then, Lemma 4 guarantees $C_{i j}(v ; \beta, \varepsilon) \in \mathbf{C}^{-}$for all $\beta \leq \bar{\beta}(\varepsilon)$, and Lemma 3 yields the result that the economy satisfies PAM.

Proof of Proposition 5. By Lemmas 3 and 4, we obtain PAM whenever $c_{i}(\beta, \varepsilon) \geq c_{j}(\beta, \varepsilon)$ for all $\langle i, j\rangle$. What remains to be shown is that the latter condition holds whenever (i) or (ii) is satisfied.

(i) Under the assumption $\varepsilon_{J}(\beta) \leq \varepsilon_{I}(\beta)$, by Lemma $5, c_{I}(\beta, \varepsilon)$ is increasing and $c_{J}(\beta, \varepsilon)$ is decreasing in $\varepsilon$ for all $\varepsilon \in\left[\varepsilon_{0}, \varepsilon_{I}(\beta)\right]$. This implies that $c_{I}(\beta, \varepsilon) \geq c_{J}(\beta, \varepsilon)$ holds for all such $\varepsilon$.

(ii) Similarly, under the assumption $\varepsilon_{I}(\beta) \leq \varepsilon_{J}(\beta)$, by Lemma 5, we know that $c_{I}(\beta, \varepsilon)$ is increasing and $c_{J}(\beta, \varepsilon)$ is decreasing in $\varepsilon$ for all $\varepsilon \in\left[\varepsilon_{I}(\beta), \varepsilon_{0}\right]$. Therefore, $c_{I}(\beta, \varepsilon) \geq$ $c_{J}(\beta, \varepsilon)$ holds for all such $\varepsilon$.

Finally, using Lemma 5 again, we know that $c_{i}(\beta, \varepsilon) \geq c_{j}(\beta, \varepsilon)$ holds for all couples $\langle i, j\rangle$ whenever $c_{I}(\beta, \varepsilon) \geq c_{J}(\beta, \varepsilon)$. 


\section{B The relevant PCs}

Lemma 6. There exists a threshold $\tilde{\varepsilon}_{i j}(\beta)$ such that $\forall \varepsilon \leq \tilde{\varepsilon}_{i j}(\beta)$ the woman's relevant PC is (4). Further, $\tilde{\varepsilon}_{i j}(\beta)>0$, and if the woman is less risk averse than the man, then $\tilde{\varepsilon}_{i j}(\beta)=1$.

Proof. First, for $\varepsilon \leq 0$ the correlation between the incomes of spouses is non-positive, and each spouse makes a positive net transfer in his/her high-income state. In particular, the woman makes a transfer in state $\underline{s}$, and hence her relevant PC is (4). Second, for $\varepsilon=1$ there is only aggregate risk, which can only be shared by spouses with different risk aversions. Further, the less risk-averse spouse shall bear more consumption risk. This implies that if the man is less risk averse than the woman, he makes a positive net transfer in state $\underline{s}$ and the woman's relevant PC is no longer (4).

What remains to be shown is that the two autarky ratios of marginal utilities, $x(j, M)$ and $x(j, W)$ are equal for at most one value of $\varepsilon$. The man's autarky ratio of marginal utilities, $x(j, M)$, is not changing with $\varepsilon$. The woman's is

$$
x(i, W)=\frac{u_{c}(i, \bar{w})}{u_{c}(i, \underline{w})}=\frac{u_{c}(i, 1+\varepsilon y)}{u_{c}(i, 1-\varepsilon y)},
$$

which is decreasing with $\varepsilon$, and the result follows.

In addition, we have the following comparative statics results for the threshold $\tilde{\varepsilon}_{i j}(\beta)$.

Lemma 7. (i) Take the man's preferences as given. The threshold $\tilde{\varepsilon}_{\cdot j}(\beta)$ decreases with the woman's risk aversion.

(ii) Take the woman's preferences as given. The threshold $\tilde{\varepsilon}_{i \cdot}(\beta)$ increases with the man's risk aversion.

Proof. (i) Let $\tilde{\varepsilon}_{i^{\prime} j}(\beta)$ denote the threshold for Ms $i^{\prime}$, which is characterised by $x\left(i^{\prime}, W\right)=$ $x(j, M)$. Now consider a more risk-averse woman, Ms $i^{\prime \prime}$, with $u\left(i^{\prime \prime}, \cdot\right)=\phi\left(u\left(i^{\prime}, \cdot\right)\right)$ and $\phi()$ increasing and strictly concave. By Lemma 6 , we have $\tilde{\varepsilon}_{i j}(\beta)>0, \forall\langle i, j\rangle$. Then, since $\phi^{\prime}()$ is decreasing, we obtain

$$
x\left(i^{\prime}, W\right)=\frac{u_{c}\left(i^{\prime}, 1+\tilde{\varepsilon}_{i^{\prime} j}(\beta) y\right)}{u_{c}\left(i^{\prime}, 1-\tilde{\varepsilon}_{i^{\prime} j}(\beta) y\right)}>\frac{\phi^{\prime}\left(u\left(i^{\prime}, 1+\tilde{\varepsilon}_{i^{\prime} j}(\beta) y\right)\right) u_{c}\left(i^{\prime}, 1+\tilde{\varepsilon}_{i^{\prime} j}(\beta) y\right)}{\phi^{\prime}\left(u\left(i^{\prime}, 1-\tilde{\varepsilon}_{i^{\prime} j}(\beta) y\right)\right) u_{c}\left(i^{\prime}, 1-\tilde{\varepsilon}_{i^{\prime} j}(\beta) y\right)}=x\left(i^{\prime \prime}, W\right) .
$$

Suppose first that $\tilde{\varepsilon}_{i^{\prime} j}=1$. The result obtains trivially, since $\tilde{\varepsilon}_{i^{\prime \prime} j}$ cannot exceed 1 by assumption. In the alternative case, i.e., when $\tilde{\varepsilon}_{i^{\prime} j}<1$, the above condition implies $x\left(i^{\prime \prime}, W\right)<$ $x(j, M)$. Therefore, the relevant PC for Ms $i^{\prime \prime}$ cannot be (4), which proves $\tilde{\varepsilon}_{i^{\prime \prime} j}<\tilde{\varepsilon}_{i^{\prime} j}$.

(ii) Applying an analogous argument to $x\left(j^{\prime}, M\right)$ and $x\left(j^{\prime \prime}, M\right)$, where $\mathrm{Mr} j^{\prime \prime}$ is more risk averse than $\mathrm{Mr} j^{\prime}$, as before, completes the proof. 
Lemma 7 first implies that as $\varepsilon$ increases the first couple for which the woman's relevant PC may no longer be (4) is the couple $\left\langle i^{\prime \prime}, j^{\prime}\right\rangle$. Second, the last couple to switch, which may occur if both women are more risk averse than both men, is the couple $\left\langle i^{\prime}, j^{\prime \prime}\right\rangle$. Finally, the second couple to switch may be either $\left\langle i^{\prime}, j^{\prime}\right\rangle$, i.e., $\tilde{\varepsilon}_{i^{\prime} j^{\prime}}<\tilde{\varepsilon}_{i^{\prime \prime} j^{\prime \prime}}$, or $\left\langle i^{\prime \prime}, j^{\prime \prime}\right\rangle$, i.e., $\tilde{\varepsilon}_{i^{\prime \prime}, j^{\prime \prime}}<\tilde{\varepsilon}_{i^{\prime} j^{\prime}}$.

\section{Algorithm to find stable matchings and determine their assortativity with dynamic contacts}

We assume for simplicity that any couple who shares risk efficiently does so for a unique relative Pareto weight in the long run. The extension to the case where some couples share risk efficiently for an interval of weights is immediate. To fix ideas, we assume that the woman makes a positive net transfer in state $\underline{s}$ for all couples. It is straightforward to consider alternatives.

Let $\alpha_{\pi}=(\pi-\pi \beta) /(1-\pi \beta)$ and $\alpha_{1-\pi}=[(1-\pi)-(1-\pi) \beta] /[1-(1-\pi) \beta]$. Further, let $\underline{c}(i, j)(\bar{c}(i, j))$ denote the long-run consumption of Ms $i$ in state $\underline{s}(\bar{s})$, which is independent of $v$, and let $U_{i}^{L R}(\underline{s}, j)=(1-\beta) u(i, \underline{c}(i, j))+\beta[(1-\pi) u(i, \bar{c}(i, j))+\pi u(i, \underline{c}(i, j))]$ denote the expected long-run per-period utility for Ms $i$ sharing risk with Mr $j$ given that the first constraint to bind is the one in state $\underline{s}$, and similarly for the other state and for $\operatorname{Mr} j$.

Step 0. Specify endowment processes, utility functions, and $\beta$.

Step 1. Compute the long-run allocation for each potential couple. This involves solving a system of two equations (the two relevant PCs) with two unknowns, consumption by Ms $i$ in state $\bar{s}$ and in state $\underline{s}$.

Step 2. Choose $v^{\prime}$, making sure that at least one feasible contract can provide this utility to $\operatorname{Mr} j^{\prime}$ when he share risk with Ms $i^{\prime}$.

Step 3. Compute $\hat{c}\left(i, j^{\prime}, v^{\prime}\right), i \in\left\{i^{\prime}, i^{\prime \prime}\right\}$ : use

$$
\alpha_{\pi} u\left(j, \underline{z}-\hat{c}\left(i, j^{\prime}, v^{\prime}\right)\right)+\left(1-\alpha_{\pi}\right) U_{j^{\prime}}^{L R}(\bar{s}, i)=v^{\prime}
$$

where we have computed the infinite sum and simplified to get this expression for the expected per-period utility of $\operatorname{Mr} J$, and verify that $\hat{c}\left(i, j^{\prime}, v^{\prime}\right)>\underline{c}\left(i, j^{\prime}, v^{\prime}\right)$; or use

$$
\alpha_{1-\pi} u\left(j, \bar{z}-\hat{c}\left(i, j^{\prime}, v^{\prime}\right)\right)+\left(1-\alpha_{\pi}\right) U_{j^{\prime}}^{L R}(\underline{s}, i)=v^{\prime}
$$

and verify that $\hat{c}\left(i, j^{\prime}, v^{\prime}\right)<\bar{c}\left(i, j^{\prime}, v^{\prime}\right)$. Note that only one or neither of the two conditions will hold for a given $v^{\prime}$. (If neither holds, then there is no transition.)

Step 4. Compute the per-period utility for Ms $i^{\prime \prime}$ sharing risk with $\operatorname{Mr} j^{\prime}$ : if $\hat{c}\left(i^{\prime \prime}, j^{\prime}, v^{\prime}\right)>$ $\underline{c}\left(i^{\prime \prime}, j^{\prime}\right)$, then

$$
\mathbb{E} u\left(i^{\prime \prime}, j^{\prime}, v^{\prime}\right)=\alpha_{\pi} u\left(i^{\prime \prime}, \hat{c}\left(i^{\prime \prime}, j^{\prime}, v^{\prime}\right)\right)+\left(1-\alpha_{\pi}\right) U_{i^{\prime \prime}}^{L R}\left(\bar{s}, j^{\prime}\right)
$$


and if $\hat{c}\left(i^{\prime \prime}, j^{\prime}, v^{\prime}\right)<\bar{c}\left(i^{\prime \prime}, j^{\prime}\right)$, then

$$
\mathbb{E} u\left(i^{\prime \prime}, j^{\prime}, v^{\prime}\right)=\alpha_{1-\pi} u\left(i^{\prime \prime}, \hat{c}\left(i^{\prime \prime}, j^{\prime}, v^{\prime}\right)\right)+\left(1-\alpha_{1-\pi}\right) U_{i^{\prime \prime}}^{L R}\left(\underline{s}, j^{\prime}\right) .
$$

Step 5. Compute $v^{\prime \prime}$ such that Ms $i^{\prime}$ is indifferent between the two potential partners: if $\hat{c}\left(i^{\prime}, j^{\prime}, v^{\prime}\right)>\underline{c}\left(i^{\prime}, j^{\prime}\right)$, then $v^{\prime \prime}$ is implicitly given by

$$
\alpha_{\pi} u\left(i^{\prime}, \hat{c}\left(i^{\prime}, j^{\prime}, v^{\prime}\right)\right)+\left(1-\alpha_{\pi}\right) U_{i^{\prime}}^{L R}\left(\bar{s}, j^{\prime}\right)=\alpha_{\pi} u\left(i^{\prime}, \hat{c}\left(i^{\prime}, j^{\prime \prime}, v^{\prime \prime}\right)\right)+\left(1-\alpha_{\pi}\right) U_{i^{\prime}}^{L R}\left(\bar{s}, j^{\prime \prime}\right),
$$

and verify that $\hat{c}\left(i^{\prime}, j^{\prime \prime}, v^{\prime \prime}\right)>\underline{c}\left(i^{\prime}, j^{\prime \prime}\right)$; and if $\hat{c}\left(i^{\prime}, j^{\prime}, v^{\prime}\right)<\bar{c}\left(i^{\prime}, j^{\prime}\right)$, then $v^{\prime \prime}$ is implicitly given by

$$
\alpha_{1-\pi} u\left(i^{\prime}, \hat{c}\left(i^{\prime}, j^{\prime}, v^{\prime}\right)\right)+\left(1-\alpha_{1-\pi}\right) U_{i^{\prime}}^{L R}\left(\underline{s}, j^{\prime}\right)=\alpha_{\pi} u\left(i^{\prime}, \hat{c}\left(i^{\prime}, j^{\prime \prime}, v^{\prime \prime}\right)\right)+\left(1-\alpha_{\pi}\right) U_{i^{\prime}}^{L R}\left(\underline{s}, j^{\prime \prime}\right),
$$

and verify that $\hat{c}\left(i^{\prime}, j^{\prime \prime}, v^{\prime \prime}\right)<\bar{c}\left(i^{\prime}, j^{\prime \prime}, v^{\prime \prime}\right)$.

Step 6. Compute $\hat{c}\left(i^{\prime \prime}, j^{\prime \prime}, v^{\prime \prime}\right)$ : use

$$
\alpha_{\pi} u\left(j, \underline{z}-\hat{c}\left(i^{\prime \prime}, j^{\prime \prime}, v^{\prime \prime}\right)\right)+\left(1-\alpha_{\pi}\right) U_{j^{\prime \prime}}^{L R}\left(\bar{s}, i^{\prime \prime}\right)=v^{\prime \prime}
$$

and verify that $\hat{c}\left(i^{\prime \prime}, j^{\prime \prime}, v^{\prime \prime}\right)>\underline{c}\left(i^{\prime \prime}, j^{\prime \prime}\right)$; or use

$$
\alpha_{1-\pi} u\left(j, \bar{z}-\hat{c}\left(i^{\prime \prime}, j^{\prime \prime}, v^{\prime \prime}\right)\right)+\left(1-\alpha_{\pi}\right) U_{j^{\prime \prime}}^{L R}\left(\underline{s}, i^{\prime \prime}\right)=v^{\prime \prime}
$$

and verify that $\hat{c}\left(i^{\prime \prime}, j^{\prime \prime}, v^{\prime \prime}\right)<\bar{c}\left(i^{\prime \prime}, j^{\prime \prime}\right)$. Note that only one or neither of the two conditions will hold for a given $v^{\prime \prime}$.

Step 7. Compute the per-period utility for Ms $i^{\prime \prime}$ sharing risk with $\operatorname{Mr} j^{\prime \prime}$ : if $\hat{c}\left(i^{\prime \prime}, j^{\prime \prime}, v^{\prime \prime}\right)>$ $\underline{c}\left(i^{\prime \prime}, j^{\prime \prime}\right)$, then

$$
\mathbb{E} u\left(i^{\prime \prime}, j^{\prime \prime}, v^{\prime \prime}\right)=\alpha_{\pi} u\left(i^{\prime \prime}, \hat{c}\left(i^{\prime \prime}, j^{\prime \prime}, v^{\prime \prime}\right)\right)+\left(1-\alpha_{\pi}\right) U_{i^{\prime \prime}}^{L R}\left(\bar{s}, j^{\prime \prime}\right)
$$

and if $\hat{c}\left(i^{\prime \prime}, j^{\prime \prime}, v^{\prime \prime}\right)<\bar{c}\left(i^{\prime \prime}, j^{\prime \prime}\right)$, then

$$
\mathbb{E} u\left(i^{\prime \prime}, j^{\prime \prime}, v^{\prime \prime}\right)=\alpha_{1-\pi} u\left(i^{\prime \prime}, \hat{c}\left(i^{\prime \prime}, j^{\prime \prime}, v^{\prime \prime}\right)\right)+\left(1-\alpha_{1-\pi}\right) U_{i^{\prime \prime}}^{L R}\left(\underline{s}, j^{\prime \prime}\right) .
$$

Step 8. Compare $\mathbb{E} u\left(i^{\prime \prime}, j^{\prime \prime}, v^{\prime \prime}\right)$ and $\mathbb{E} u\left(i^{\prime \prime}, j^{\prime}, v^{\prime}\right)$. If $\mathbb{E} u\left(i^{\prime \prime}, j^{\prime \prime}, v^{\prime \prime}\right) \geq \mathbb{E} u\left(i^{\prime \prime}, j^{\prime}, v^{\prime}\right)$, then we have found a stable matching which is positive assortative. If $\mathbb{E} u\left(i^{\prime \prime}, j^{\prime \prime}, v^{\prime \prime}\right) \leq \mathbb{E} u\left(i^{\prime \prime}, j^{\prime}, v^{\prime}\right)$, then we have found a stable matching which is negative assortative. 